\title{
Elaborating Dependent (Co)pattern Matching
}

\author{
JESPER COCKX and ANDREAS ABEL, Chalmers and Gothenburg University, Sweden
}

In a dependently typed language, we can guarantee correctness of our programs by providing formal proofs. To check them, the typechecker elaborates these programs and proofs into a low level core language. However, this core language is by nature hard to understand by mere humans, so how can we know we proved the right thing? This question occurs in particular for dependent copattern matching, a powerful language construct for writing programs and proofs by dependent case analysis and mixed induction/coinduction. A definition by copattern matching consists of a list of clauses that are elaborated to a case tree, which can be further translated to primitive eliminators. In previous work this second step has received a lot of attention, but the first step has been mostly ignored so far.

We present an algorithm elaborating definitions by dependent copattern matching to a core language with inductive datatypes, coinductive record types, an identity type, and constants defined by well-typed case trees To ensure correctness, we prove that elaboration preserves the first-match semantics of the user clauses. Based on this theoretical work, we reimplement the algorithm used by Agda to check left-hand sides of definitions by pattern matching. The new implementation is at the same time more general and less complex, and fixes a number of bugs and usability issues with the old version. Thus we take another step towards the formally verified implementation of a practical dependently typed language.

CCS Concepts: • Theory of computation $\rightarrow$ Type theory; $\bullet$ Software and its engineering $\rightarrow$ Functional languages; Patterns;

Additional Key Words and Phrases: Dependent types, Dependent pattern matching, Copatterns, Agda

ACM Reference Format:

Jesper Cockx and Andreas Abel. 2018. Elaborating Dependent (Co)pattern Matching. Proc. ACM Program. Lang. 2, ICFP, Article 75 (September 2018), 30 pages. https://doi.org/10.1145/3236770

\section{INTRODUCTION}

Dependently typed functional languages such as Agda [2017], Coq [INRIA 2017], Idris [2013], and Lean [de Moura et al. 2015] combine programming and proving into one language, so they should be at the same time expressive enough to be useful and simple enough to be sound. These apparently contradictory requirements are addressed by having two languages: a high-level surface language that focuses on expressivity and a small core language that focuses on simplicity. The main role of the typechecker is to elaborate the high-level surface language into the low-level core.

Since the difference between the surface and core languages can be quite large, the elaboration process can be, well, elaborate. If there is an error in the elaboration process, our program or proof may still be accepted by the system but its meaning is not what was intended [Pollack 1998]. In particular, the statement of a theorem may depend on the correct behavior of some defined function, so if something went wrong in the elaboration of these definitions, the theorem statement may not be what it seems. As an extreme example, we may think we have proven an interesting

Authors' address: Jesper Cockx, cockx@chalmers.se; Andreas Abel, andreas.abel@gu.se, Chalmers and Gothenburg University, Department of Computer Science and Engineering, Rännvägen 6b, Gothenburg, 41296, Sweden.

This work is licensed under a Creative Commons Attribution 4.0 International License.

(c) 2018 Copyright held by the owner/author(s).

2475-1421/2018/9-ART75

https://doi.org/10.1145/3236770

Proc. ACM Program. Lang., Vol. 2, No. ICFP, Article 75. Publication date: September 2018. 
theorem when in fact, we have only proven something trivial. This may be detected in a later phase when trying to use this proof, or it may not be detected at all. Unfortunately, there is no bulletproof way to avoid such problems: each part of the elaboration process has to be verified independently to make sure it produces something sensible.

One important part of the elaboration process is the elaboration of definitions by dependent pattern matching [Coquand 1992]. Dependent pattern matching provides a convenient high-level interface to the low-level constructions of case splitting, structural induction, and specialization by unification. The elaboration of dependent pattern matching goes in two steps: first the list of clauses given by the user is translated to a case tree, and then the case tree is further translated to a term that only uses the primitive datatype eliminators. ${ }^{1}$ The second step has been studied in detail and is known to preserve the semantics of the case tree precisely [Cockx 2017; Goguen et al. 2006]. In contrast, the first step has received much less attention.

The goal of this paper is to formally describe an elaboration process of definitions by dependent pattern matching to a well-typed case tree for a realistic dependently typed language. Compared to the elaboration processes described by Norell [2007] and Sozeau [2010], we make the following improvements:

- We include both pattern and copattern matching.

- We are more flexible in the placement of forced patterns.

- We prove that the translation preserves the first-match semantics of the user clauses.

We discuss each of these improvements in more detail below.

Copatterns. Copatterns provide a convenient way to define and reason about infinite structures such as streams [Abel et al. 2013]. They can be nested and mixed with regular patterns. Elaboration of definitions by copattern matching has been studied for simply typed languages by Setzer et al. [2014], but so far the combination of copatterns with general dependent types has not been studied in detail, even though it has already been implemented in Agda.

One complication when dealing with copatterns in a dependently typed language is that the type of a projection can depend on the values of the previous projections. For example, define the coinductive type CoNat of possibly infinite natural numbers by the two projections iszero : Bool and pred : iszero $\equiv_{\text {Bool }}$ false $\rightarrow$ CoNat. We use copatterns to define the co-natural number cozero:

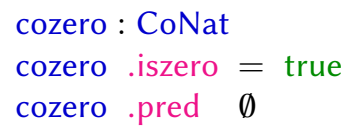

Here the constant cozero is being defined with the field iszero equal to true (and no value for pred).

To refute the proof of cozero .iszero $\equiv_{\text {Bool }}$ false with an absurd pattern $\emptyset$, the typechecker needs to know already that cozero .iszero $=$ true, so it needs to check the clauses in the right order.

This example also shows that with mixed pattern/copattern matching, some clauses can have more arguments than others, so the typechecker has to deal with variable arity. This means that we need to consider introducing a new argument as an explicit node in the constructed case tree.

Flexible placement of forced patterns. When giving a definition by dependent pattern matching that involves forced patterns (also called presupposed terms [Brady et al. 2003] or inaccessible

\footnotetext{
${ }^{1}$ In Agda, case trees are part of the core language so the second step is skipped in practice, but it is still important to know that it could be done in theory.
} 
patterns [Norell 2007] or, in Agda, dot patterns), there are often multiple positions where to place them. For example, in the proof of symmetry of equality

$$
\begin{aligned}
& \operatorname{sym}:(x y: A) \rightarrow x \equiv_{A} y \rightarrow y \equiv_{A} x \\
& \operatorname{sym} x\lfloor x\rfloor \text { refl }=\operatorname{refl}
\end{aligned}
$$

it should not matter if we instead write sym $\lfloor x\rfloor x$ refl = refl. In fact, we even allow the apparently non-linear definition sym $x x$ refl $=$ refl.

Our elaboration algorithm addresses this by treating forced patterns as laziness annotations: they guarantee that the function will not match against a certain argument. This allows the user to be free in the placement of the forced patterns. For example, it is always allowed to write zero instead

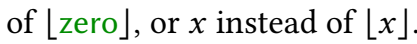

With our elaboration algorithm, it is easy to extend the pattern syntax with forced constructor patterns such as โsuc」 $n$ (Brady et al. [2003]'s presupposed-constructor patterns). These allow the user to annotate that the function should not match on the argument but still bind some of the arguments of the constructor.

Preservation of first-match semantics. Like Augustsson [1985] and Norell [2007], we allow the clauses of a definition by pattern matching to overlap and use the first-match semantics in the construction of the case tree. For example, when constructing a case tree from the definition

$\max : \mathbb{N} \rightarrow \mathbb{N} \rightarrow \mathbb{N}$
$\max$ zero $y=y$
$\max x \quad$ zero $=x$
$\max (\operatorname{suc} x)(\operatorname{suc} y)=\operatorname{suc}(\max x y)$

we do not get $\max x$ zero $=x$ but only $\max \left(\operatorname{suc} x^{\prime}\right)$ zero $=$ suc $x^{\prime}$. This makes a difference for dependent type checking where we evaluate open terms with free variables like $x$. In this paper we provide a proof that the translation from a list of clauses to a case tree preserves the first-match semantics of the clauses. More precisely, we prove that if the arguments given to a function match a clause and all previous clauses produce a mismatch, ${ }^{2}$ then the case tree produced by elaborating the clauses also computes for the given arguments and the result is the same as the one given by the clause.

\section{Contributions.}

- We present a dependently typed core language with inductive datatypes, coinductive record types and an identity type. The language is focused [Andreoli 1992; Krishnaswami 2009; Zeilberger 2008]: terms of our language correspond to the non-invertible rules to introduce and eliminate these types, while the invertible rules constitute case trees.

- We are the first to present a coverage checking algorithm for fully dependent copatterns. Our algorithm desugars deep copattern matching to well-typed case trees in our core language.

- We prove correctness: if the desugaring succeeds, then the behavior of the case tree corresponds precisely to the first-match semantics of the given clauses.

- We have implemented a new version of the algorithm used by Agda for checking the lefthand sides of a definition by dependent (co)pattern matching, which has been released as part of Agda 2.5.4. ${ }^{3}$ At the time of writing the effort to remodel the elaboration to a case tree according to the theory presented in this paper is still ongoing, but our work so far has already uncovered and fixed multiple issues in the old implementation [Agda issue

\footnotetext{
${ }^{2}$ Note that, in the example, the open term max $x$ zero does not produce a mismatch with the first clause since it could match if variable $x$ was replaced by zero. In the first-match semantics, evaluation of max $x$ zero is stuck.

${ }^{3}$ Agda 2.5.4 released on 2018/06/02, changelog: https://hackage.haskell.org/package/Agda-2.5.4/changelog.
} 
2017a,b,c,d, 2018a,b]. Our algorithm could also be used by other implementations of dependent pattern matching such as the Equations package for Coq [Sozeau 2010], Idris [2013], and Lean [de Moura et al. 2015].

This paper was born out of a practical need that arose while reimplementing the elaboration algorithm for Agda: it was not clear to us what exactly we wanted to implement, and we did not find sufficiently precise answers in the existing literature. Our main goal in this paper is therefore to give a precise description of the language, the elaboration algorithm, and the high-level properties we expect them to have. This also means we do not focus on fully developing the metatheory of the language or giving detailed proofs for all the basic properties one would expect.

We start by introducing definitions by dependent (co)pattern matching and our elaboration algorithm to a case tree by a number of examples in Sect. 2. We then describe our core language in Sect. 3: the syntax, the rules for typing and equality, and the evaluation rules. In Sect. 4 we give the syntax and rules for case trees, and prove that a function defined by a well-typed case tree satisfies type preservation and coverage. Finally, in Sect. 5 we describe the rules for elaborating a definition by dependent (co)pattern matching to a well-typed case tree, and prove that this translation preserves the computational meaning of the given clauses. Sect. 6 discusses related work, and Sect. 7 concludes.

This is the short version of this article with most proofs omitted. A longer version with proofs can be found on the first author's website. ${ }^{4}$

\section{ELABORATING DEPENDENT (CO)PATTERN MATCHING BY EXAMPLE}

Before we move on to the general description of our core language and the elaboration process, we give some examples of definitions by (co)pattern matching and how our algorithm elaborates them to a case tree. The elaboration works on a configuration $\Gamma \vdash P \mid u: C$ consisting of:

- A context $\Gamma$, i.e. a list of variables annotated with types. Initially $\Gamma$ is the empty context $\epsilon$.

- The current target type $C$. This type may depend on variables bound in $\Gamma$. Initially $C$ is the type of the function being defined.

- A representation of the left-hand side $u$. In the end $u$ should have type $C$ in context $\Gamma$. Initially $u$ is the function being defined itself.

- A list of partially deconstructed user clauses $P$. Initially these are the clauses as written by the user.

These four pieces of data together describe the current state of elaborating the definition.

The elaboration algorithm transforms this state step by step until the user clauses are deconstructed completely. In the examples below, we annotate each step with a label such as SpLITCON or INTRO, linking it to the general rules given in Sect. 5.

Example 1. Let us define a function $\max : \mathbb{N} \rightarrow \mathbb{N} \rightarrow \mathbb{N}$ by pattern matching as in the introduction (3). The initial configuration is $\vdash P_{0} \mid \max : \mathbb{N} \rightarrow \mathbb{N} \rightarrow \mathbb{N}$ where

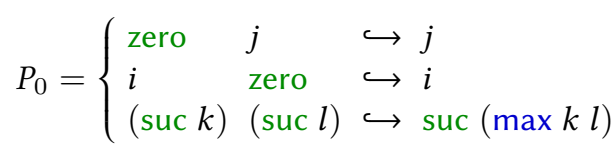

\footnotetext{
${ }^{4}$ https://jesper.sikanda.be/files/elaborating-dependent-copattern-matching.pdf
} 
The first operation we need is to introduce a new variable $m$ (rule INTRO). It transforms the initial problem into $(m: \mathbb{N}) \vdash P_{1} \mid \max m: \mathbb{N} \rightarrow \mathbb{N}$ where

$$
P_{1}=\left\{\begin{array}{lll}
{[m / ? \text { zero }]} & j & \hookrightarrow j \\
{[m / ? i]} & \text { zero } & \hookrightarrow i \\
{[m / ? \text { suc } k]} & (\operatorname{suc} l) & \hookrightarrow \text { suc }(\max k l)
\end{array}\right.
$$

This operation strips the first user pattern from each clause and replaces it by a constraint $m / ? p$ that it should be equal to the newly introduced variable $m$. We write these constraints between brackets in front of each individual clause.

The next operation we need is to perform a case analysis on the variable $m$ (rule SplitCon). ${ }^{5}$ This transforms the problem into two subproblems $\vdash P_{2} \mid \max$ zero $: \mathbb{N} \rightarrow \mathbb{N}$ and $(p: \mathbb{N}) \vdash$ $P_{3} \mid \max (\operatorname{suc} p): \mathbb{N} \rightarrow \mathbb{N}$ where

$$
\begin{aligned}
& P_{2}=\left\{\begin{array}{lll}
{[\text { zero } / \text { zero }]} & j & \hookrightarrow j \\
{[\text { zero } / ?]} & \text { zero } & \hookrightarrow i \\
{[\text { zero } / \text { suc } k]} & (\operatorname{suc} l) & \hookrightarrow \text { suc }(\max k l)
\end{array}\right. \\
& P_{3}=\left\{\begin{array}{lll}
{[\operatorname{suc} p / ? \text { zero }]} & j & \hookrightarrow j \\
{[\operatorname{suc} p / ?} & \text { zero } & \hookrightarrow i \\
{[\operatorname{suc} p / ? \text { suc } k]} & (\operatorname{suc} l) & \hookrightarrow \text { suc }(\max k l)
\end{array}\right.
\end{aligned}
$$

We simplify the constraints, removing those clauses with absurd constraints:

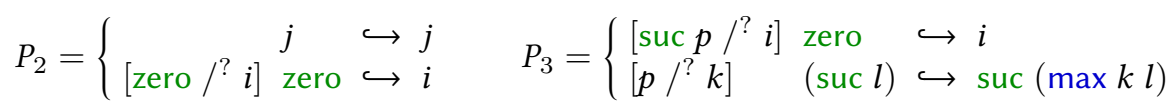

We continue applying these operations INTRO and SPLITCON (introducing a new variable and case analysis on a variable) until the first clause has no more user patterns and no more constraints where the left-hand side is a constructor. For example, for $P_{2}$ we get after one more introduction step $(n: \mathbb{N}) \vdash P_{4} \mid \max$ zero $n: \mathbb{N}$ where

$$
P_{4}= \begin{cases}{[n / ? j]} & \hookrightarrow j \\ {[\text { zero } / ? i, n / ? \text { zero }]} & \hookrightarrow i\end{cases}
$$

We solve the remaining constraint in the first clause by instantiating $j:=n$. This means we are done and we have max zero $n=j[n / j]=n$ (rule DonE). Similarly, elaborating the lhs prob$\operatorname{lem}(p: \mathbb{N}) \vdash P_{3} \mid \max (\operatorname{suc} p): \mathbb{N} \rightarrow \mathbb{N}$ (with rules Intro, SplitCon, and Done) gives us $\max (\operatorname{suc} p)$ zero $=\operatorname{suc} p$ and $\max (\operatorname{suc} p)(\operatorname{suc} q)=\operatorname{suc}(\max p q)$.

We record the operations used when elaborating the clauses in a case tree. Our syntax for case trees is close to the normal term syntax in other languages: $\lambda x$. for introducing a new variable and case $_{x}\{\}$ for a case split. For max, we get the following case tree:

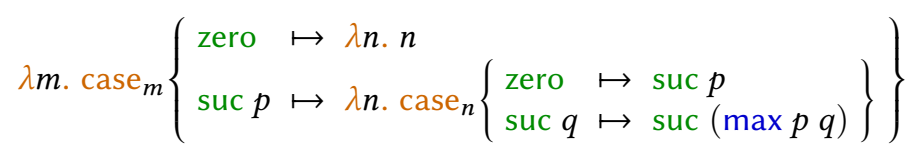

Example 2. Next we take a look at how to elaborate definitions using copatterns. For the cozero example (1), we have the initial configuration $\vdash P_{0} \mid$ cozero : CoNat where:

$$
P_{0}=\left\{\begin{array}{l}
\text {.iszero } \hookrightarrow \text { true } \\
. \text { pred } \emptyset \hookrightarrow \text { impossible }
\end{array}\right.
$$

\footnotetext{
${ }^{5}$ At this point we could also introduce the variable for the second argument of max, the elaboration algorithm is free to choose either option.
} 
Here we need a new operation to split on the result type CoNat (rule Cosplit). This produces two subproblems $\vdash P_{1} \mid$ cozero .iszero and $\vdash P_{2} \mid$ cozero .pred : cozero .iszero $\equiv_{\text {Bool }}$ false $\rightarrow$ CoNat where

$$
P_{1}=\left\{\hookrightarrow \text { true } \quad P_{2}=\{\emptyset \hookrightarrow \text { impossible }\right.
$$

The first problem is solved immediately with cozero .iszero = true (rule DonE). In the second problem we introduce the variable $x$ : cozero .iszero $\equiv_{\text {Bool }}$ false (rule INTro) and note that

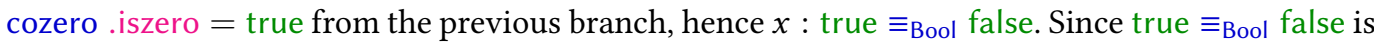
an empty type (technically, since unification of true with false results in a conflict), we can perform a case split on $x$ with zero cases (rule SplitEmpty), solving the problem.

In the resulting case tree, the syntax for a split on the result type is record \{\} , with one subtree for each field of the record type:

$$
\operatorname{record}\left\{\begin{aligned}
\text { iszero } & \mapsto \text { true } \\
\text { pred } & \mapsto \lambda x . \operatorname{case}_{x}\{\}
\end{aligned}\right\}
$$

For the next examples, we omit the details of the elaboration process and only show the definition by pattern matching and the resulting case tree.

Example 3. Consider the type CStream of C streams: potentially infinite streams of numbers that end on a zero. We define this as a record where the tail field has two extra arguments enforcing that we can only take the tail if the head is suc $m$ for some $m$.

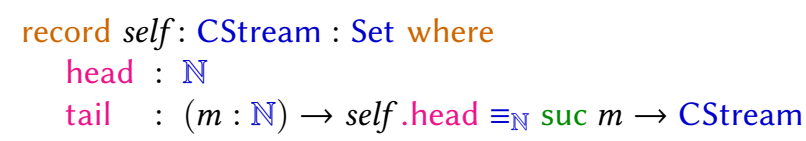

Here, the name self is bound to the current record instance, allowing later projections to depend on prior projections.

Now consider the function countdown that creates a $\mathrm{C}$ stream counting down from a given number $n$ :

$$
\begin{aligned}
& \text { countdown : } \mathbb{N} \rightarrow \text { CStream } \\
& \text { countdown } n \text {.head }=n \\
& \text { countdown zero tail } m \emptyset \\
& \text { countdown }(\text { suc } m \text { ) .tail } m \text { refl }=\text { countdown } m
\end{aligned}
$$

Our elaboration algorithm applies rules Intro, Cosplit, SplitCon, SplitEmpty, SplitEQ, and DonE in sequence to translate this definition to the following case tree:

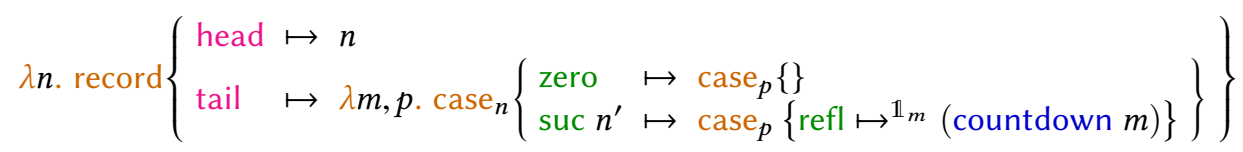

Note the extra annotation $\mathbb{1}_{m}$ after the case split on $p$ : suc $m \equiv_{\mathbb{N}}$ suc $n^{\prime}$. This is a substitution (in this case the identity substitution on $(m: \mathbb{N}))$ necessary for the evaluation rules of the case tree when matching on refl. It reflects the fact that $n^{\prime}$ went out of scope after the case split on refl : suc $n^{\prime} \equiv_{\mathbb{N}}$ suc $m$ (since unification instantiated it with $m$ ) so only the variable $m$ can still be used after this point.

Example 4. This example is based on issue \#2896 on the Agda bug tracker [Agda issue 2018b]. The problem was that Agda's old elaboration algorithm threw away a part of the pattern written by the user. This meant the definition could be elaborated to a different case tree from the one intended by the user. 
The (simplified) example consists of the following datatype $D$ and function foo:

$$
\begin{aligned}
\text { data } \mathrm{D}(m: \mathbb{N}): \text { Set where } & \text { foo }:(m: \mathbb{N}) \rightarrow \mathrm{D}(\text { suc } m) \rightarrow \mathbb{N} \\
\text { c }:(n: \mathbb{N})\left(p: n \equiv_{\mathbb{N}} m\right) \rightarrow \mathrm{D} m & \text { foo } m(\text { c (suc } n) \text { refl })=m+n
\end{aligned}
$$

The old algorithm would ignore the pattern suc $n$ in the definition of foo because it corresponds to a forced pattern after the case split on refl. Our elaboration instead produces the following case tree (using rules Intro, SplitCon, SplitEQ, and Done):

$$
\lambda m, x . \operatorname{case}_{x}\left\{\text { c } n p \mapsto \operatorname{case}_{p}\left\{\operatorname{refl} \mapsto^{\mathbb{1}_{m}}(m+m)\right\}\right\}
$$

Even though this case tree does not match on the suc constructor, it implements the same computational behavior as the clause in the definition of foo because the first argument of $\mathrm{c}$ is forced to be suc $m$ by the typing rules.

This example also shows another feature supported by our elaboration algorithm, namely that two different variables $m$ and $n$ in the user syntax may correspond to the same variable $m$ in the core syntax. In effect, $n$ is treated as a let-bound variable with value $m$.

Example 5. This example is based on issue \#2964 on the Agda bug tracker [Agda issue 2018a]. The problem was that Agda was using a too liberal version of the first-match semantics that was not preserved by the translation to a case tree. The problem occurred for the following definition:

$$
\begin{aligned}
& \mathrm{f}:(A: \text { Set }) \rightarrow A \rightarrow \text { Bool } \rightarrow\left(A \equiv_{\text {Set }} \text { Bool }\right) \rightarrow \text { Bool } \\
& \mathrm{f}\lfloor\text { Bool }\rfloor \text { true true refl }
\end{aligned}
$$

This function is elaborated (both by Agda's old algorithm and by ours) to the following case tree (using rules Intro, SplitCon, SplitEq, and Done):

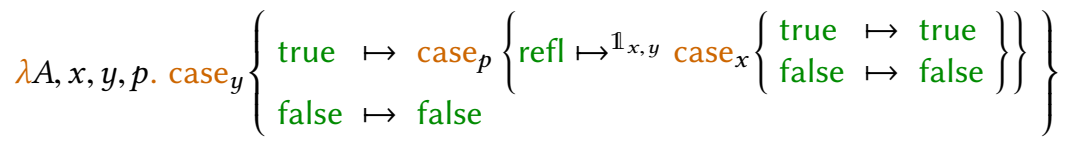

According to the (liberal) first-match semantics, we should have $f$ Bool false $y p=$ false for any $y:$ Bool and $p:$ Bool $\equiv_{\text {Set }}$ Bool, but this is not true for the case tree since evaluation gets stuck on the variable $y$. Another possibility is to start the case tree by a split on $p$ (after introducing all the variables), but this case tree still gets stuck on the variable $p$. In fact, there is no well-typed case tree that implements the first-match semantics of these clauses since we cannot perform a case split on $x: A$ before splitting on $p$.

One radical solution for this problem would be to only allow case trees where the case splits are performed in order from left to right. However, this would mean the typechecker must reject many definitions such as $\mathrm{f}$ in this example, because the type of $x$ is not known to be a datatype until the case split on $A \equiv_{\text {Set }}$ Bool. Instead we choose to keep the elaboration as it is and restrict the first-match semantics of clauses. In the example of $f$, this change means that we can only go to the second clause once all three arguments $x, y$ and $p$ are constructors, and at least one of them produces a mismatch.

\section{CORE LANGUAGE}

In this section we introduce a basic type theory for studying definitions by dependent (co)pattern matching. It has support for dependent function types, an infinite hierarchy of predicative universes, equality types, inductive datatypes and coinductive records.

To keep the work in this paper as simple as possible, we leave out many features commonly included in dependently typed languages, such as lambda expressions and inductive families of 
datatypes (other than the equality type). These features can nevertheless be encoded in our language, see Sect. 3.5 for details.

Note also that we do not include any rules for $\eta$-equality, neither for lambda expressions (which do not exist) nor for records (which can be coinductive hence do not satisfy $\eta$ ). Sect. 3.5 discusses how our language could be extended with $\eta$-rules.

\subsection{Syntax of the Core Type Theory}

Expressions of our type theory are almost identical to Agda's internal term language. All function applications are in spine-normal form, so the head symbol of an application is exposed, be it variable $x$, data $\mathrm{D}$ or record type $\mathrm{R}$, or defined function $\mathrm{f}$. We generalize applications to eliminations $e$ by including projections.$\pi$ in spines $\bar{e}$. Any expression is in weak head normal form but $\bar{e} \bar{e}$, which is computed via pattern matching (see Sect. 3.4).

$\begin{aligned} A, B, u, v::= & w \\ W, w \quad::= & (x: A) \rightarrow B \\ & \mid \begin{array}{l}\mid \\ \end{array} \quad \mathrm{D} \bar{u} \\ & \mathrm{R} \bar{u} \\ & u \equiv_{A} v \\ & x \bar{e} \\ & \mathrm{c} \bar{u} \\ & \text { refl }\end{aligned}$

weak head normal form

defined function applied to eliminations

dependent function type universe $\ell$

datatype fully applied to parameters record type fully applied to parameters equality type

variable applied to eliminations constructor fully applied to arguments proof of reflexivity

Any expression but c $\bar{u}$ or refl can be a type; the first five weak head normal forms are definitely types. Any type has in turn a type, specifically some universe Set $\ell$. Syntax is colored according to the Agda conventions: primitives and defined symbols are blue, constructors are green, and projections are pink.

$$
\begin{array}{ccr}
e::=u & \text { application } \\
\mid \quad \cdot \pi & \text { projection }
\end{array}
$$

Binary application $u e$ is defined as a partial function on the syntax: for variables and functions it is defined by $(x \bar{e}) e=x(\bar{e}, e)$ and $(\mathrm{f} \bar{e}) e=\mathrm{f}(\bar{e}, e)$ respectively, otherwise it is undefined.

Patterns are generated from variables and constructors. In addition, we have forced and absurd patterns. Since we are matching spines, we also consider projections as patterns, or more precisely, as copatterns.

$$
\begin{aligned}
& p::=x \quad \text { variable pattern } \\
& \text { | refl pattern for reflexivity proof } \\
& \text { | с } \bar{p} \quad \text { constructor pattern } \\
& \text { | \c」 }\rfloor \quad \text { forced constructor pattern }
\end{aligned}
$$

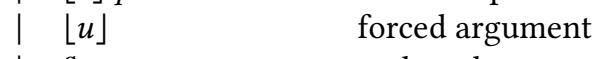

$$
\begin{aligned}
& \text { | } \emptyset \text { absurd pattern } \\
& \begin{array}{cr}
q::=p & \text { application copattern } \\
\mid \quad . \pi & \text { projection copattern }
\end{array}
\end{aligned}
$$

Forced patterns [Brady et al. 2003] appear with dependent types; they are either entirely forced arguments $\lfloor u\rfloor$, which are Agda's dot patterns, or only the constructor is forced $\lfloor c\rfloor \bar{p}$. An argument 
can be forced by a match against refl somewhere in the surrounding (co)pattern. However, sometimes we want to bind variables in a forced argument; in this case, we revert to forced constructors. Absurd patterns ${ }^{6}$ are used to indicate that the type at this place is empty, i.e. no constructor can possibly match. They are also used to indicate an empty copattern split, i.e. a copattern split on a record type with no projections. This allows us in particular to define the unique element tt of the unit record, which has no projections at all, by the clause tt $\emptyset=$ impossible.

The pattern variables $\mathrm{PV}(\bar{q})$ is the list of variables in $\bar{q}$ that appear outside forcing brackets $\lfloor\cdot\rfloor$. By removing the forcing brackets, patterns $p$ embed into terms $\lceil p\rceil$, and copatterns $q$ into eliminations $\lceil q\rceil$, except for the absurd pattern $\emptyset$.

$$
\begin{aligned}
& \lceil x\rceil=x \quad\lceil c \bar{p}\rceil=\text { c }\lceil\bar{p}\rceil \quad\lceil\lfloor u\rfloor\rceil=u \\
& \lceil\mathrm{refl}\rceil=\mathrm{refl} \quad\lceil\lfloor\mathrm{c}\rfloor \bar{p}\rceil=\mathrm{c}\lceil\bar{p}\rceil \quad\lceil. \pi\rceil=. \pi
\end{aligned}
$$

Constructors take a list of arguments whose types can depend on all previous arguments. The constructor parameters are given as a list $x_{1}: A_{1}, \ldots, x_{n}: A_{n}$ with pairwise distinct $x_{i}$ where $A_{i}$ can depend on $x_{1}, \ldots, x_{i-1}$. This list can be conceived as a cons-list, then it is called a telescope, or as a snoc-list, then we call it a context.

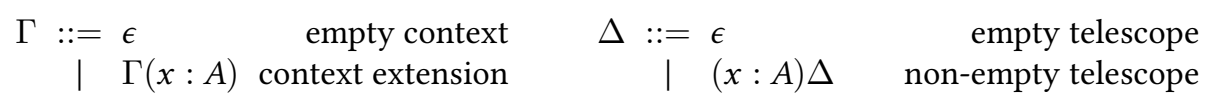

Context and telescopes can be regarded as finite maps from variables to types, and we require $x \notin \operatorname{dom}(\Gamma)$ and $x \notin \operatorname{dom}(\Delta)$ in the above grammars. We implicitly convert between contexts and telescopes, but there are still some conceptual differences. Contexts are always closed, i.e. its types only refer to variables bound prior in the same context. In contrast, we allow open telescopes whose types can also refer to some surrounding context. Telescopes can be naturally thought of as context extensions, and if $\Gamma$ is a context and $\Delta$ a telescope in context $\Gamma$ where $\operatorname{dom}(\Gamma)$ and $\operatorname{dom}(\Delta)$ are disjoint, then $\Gamma \Delta$ defined by $\Gamma \epsilon=\Gamma$ and $\Gamma((x: A) \Delta)=(\Gamma(x: A)) \Delta$ is a new valid context. We embed telescopes in the syntax of declarations, but contexts are used in typing rules exclusively.

Given a telescope $\Delta$, let $\hat{\Delta}$ be $\Delta$ without the types, i.e. the variables of $\Delta$ in order. Further, we define $\Delta \rightarrow C$ as the iterated dependent function type via $\epsilon \rightarrow C=C$ and $(x: A) \Delta \rightarrow C=$ $(x: A) \rightarrow(\Delta \rightarrow C)$.

A development in our core type theory is a list of declarations, of which there are three kinds: data type, record type, and function declarations (see Fig. 1). The input to the type checker is a list of unchecked declarations $\operatorname{decl}^{\ominus}$, and the output a list of checked declarations $\mathrm{decl}^{\oplus}$, called a signature $\Sigma$.

A data type $\mathrm{D}$ can be parameterized by telescope $\Delta$ and inhabits one of the universes Set . $_{\text {. }}$ Each of its constructors $\mathrm{c}_{\mathrm{i}}$ (although there might be none) takes a telescope $\Delta_{i}$ of arguments that can refer to the parameters in $\Delta$. The full type of $\mathrm{c}_{\mathrm{i}}$ could be $\Delta \Delta_{i} \rightarrow \mathrm{D} \hat{\Delta}$, but we never apply constructors to the data parameters explicitly.

A record type $\mathrm{R}$ can be thought of as a single constructor data type; its fields $\pi_{1}: A_{1}, \ldots, \pi_{\mathrm{n}}: A_{n}$ would be the constructor arguments. The field list behaves similar to a telescope, the type of each field can depend on the value of the previous fields. However, these values are referred to via self.$\pi_{\mathrm{i}}$ where variable self is a placeholder for the value of the whole record. ${ }^{7}$ The full type of projection $\pi_{\mathrm{i}}$ could be $\Delta($ self $: \mathrm{R} \hat{\Delta}) \rightarrow A_{i}$, but like for constructors, we do not apply a projection explicitly to the record parameters.

\footnotetext{
${ }^{6}$ Absurd patterns are written as "( )" in Agda syntax.

${ }^{7}$ self is the analogous of Java's "this", but like in Scala's "trait", the name can be chosen.
} 


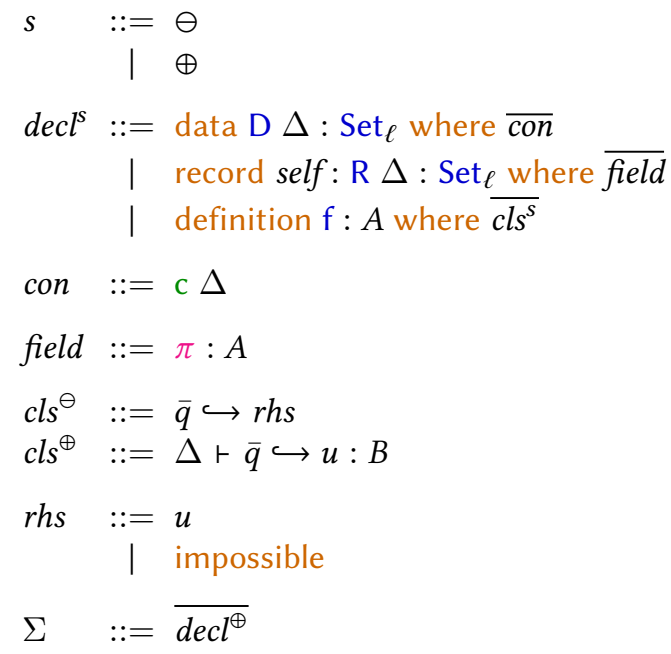

status: unchecked status: checked

datatype declaration record declaration function declaration constructor declaration field declaration unchecked clause checked clause

clause body: expression empty body for absurd pattern signature

Fig. 1. Declarations.

Even though we do not spell out the conditions for ensuring totality in this paper, like positivity, termination, and productivity checking, data types, when recursive, should be thought of as inductive types, and record types, when recursive, as coinductive types [Abel et al. 2013]. Thus, there is no dedicated constructor for records; instead, concrete records are defined by what their projections compute.

Such definitions are subsumed under the last alternative dubbed function declaration. More precisely, these are definitions by copattern matching which include record definitions. Each clause defining the constant $\mathrm{f}$ : $A$ consists of a list of copatterns $\bar{q}$ and right hand side rhs. The copatterns eliminate type $A$ into the type of the $r h s$ which is either a term $u$ or the special keyword impossible, in case one of the copatterns $q_{i}$ contains an absurd pattern $\emptyset$. The intended semantics is that if an application $\mathrm{f} \bar{e}$ matches a left hand side $\mathrm{f} \bar{q}$ with substitution $\sigma$, then $\mathrm{f} \bar{e}$ reduces to $r h s$ under $\sigma$. For efficient computation of matching, we require linearity of pattern variables for checked clauses: each variable in $\bar{q}$ occurs only once in a non-forced position.

While checking declarations, the typechecker builds up a signature $\Sigma$ of already checked declarations or parts of declarations. Checked clauses are the elaboration (sections 2 and 5) of the corresponding unchecked clauses: they are non-overlapping and supplemented by a telescope $\Delta$ holding the types of the pattern variables and the type $B$ of left and right hand side. Further, checked clauses do not contain absurd patterns.

In the signature, the last entry might be incomplete, e.g. a data type missing some constructors, a record type missing some fields, or a function missing some clauses. During checking a declaration, we might add already checked parts of the declaration, dubbed snippets, to the signature.

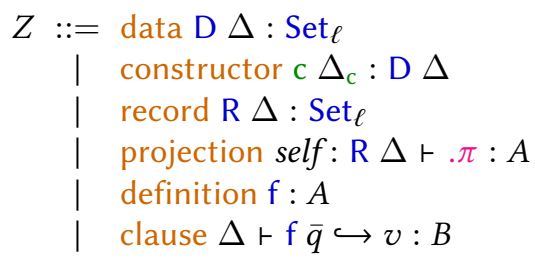

data type signature constructor signature record type signature projection signature function signature function clause 
Adding a snippet $Z$ to a signature $\Sigma$, written $\Sigma, Z$ is a always defined if $Z$ is a data or record type or function signature; in this case, the corresponding declaration is appended to $\Sigma$. Adding a constructor signature constructor c $\Delta_{\mathrm{c}}$ : D $\Delta$ is only defined if the last declaration in $\Sigma$ is (data D $\Delta$ : Set $\ell$ where $\overline{c o n}$ ) and $\mathrm{c}$ is not part of $\overline{c o n}$ yet. Analogous conditions apply when adding projection snippets. Function clauses can be added if the last declaration of $\Sigma$ is a function declaration with the same name. We trust the formal definition of $\Sigma, Z$ to the imagination of the reader. The conditions ensure that we do not add new constructors to a data type that is already complete or new fields to a completed record declaration. Such additions could destroy coverage for functions that have already been checked. Late addition of function clauses would not pose a problem, but that feature would be obsolete for our type theory anyway.

Membership of a snippet is written $Z \in \Sigma$ and a decidable property with the obvious definition. These operations on the signature will be used in the inference rules of our type theory. Since we only refer to a constructor $\mathrm{c}$ in conjunction with its data type $\mathrm{D}$, constructors can be overloaded, and likewise projections.

\subsection{Typing and Equality}

Our type theory employs the following basic typing and equality judgments, which are relative to a signature $\Sigma$.
$\Sigma+\Gamma$
$\Sigma ; \Gamma r_{\ell} \Delta$
$\Sigma ; \Gamma \vdash u: A$
$\Sigma ; \Gamma \vdash \bar{u}: \Delta$
$\Sigma ; \Gamma \mid u: A+\bar{e}: B$
$\Sigma ; \Gamma \vdash u=v: A$
$\Sigma ; \Gamma+\bar{u}=\bar{v}: \Delta$
$\Sigma ; \Gamma \mid u: A \vdash \bar{e}=\bar{e}^{\prime}: B$
context $\Gamma$ is well-formed
in context $\Gamma$, telescope $\Delta$ is well-formed and $\ell$-bounded
in context $\Gamma$, term $u$ has type $A$
in context $\Gamma$, term list $\bar{u}$ instantiates telescope $\Delta$
in context $\Gamma$, head $u$ of type $A$ is eliminated via $\bar{e}$ to type $B$
in context $\Gamma$, terms $u$ and $v$ are equal of type $A$
in context $\Gamma$, term lists $\bar{u}$ and $\bar{v}$ are equal instantiations of $\Delta$

In all these judgements, the signature $\Sigma$ is fixed, thus we usually omit it, e.g. in the inferences rules.

We further define some shorthands for type-level judgements when we do not care about the universe level $\ell$ :

$$
\begin{array}{lll}
\Sigma ; \Gamma \vdash \Delta & \Longleftrightarrow \exists \ell . \Sigma ; \Gamma \vdash \ell \Delta & \text { well-formed telescope } \\
\Sigma ; \Gamma \vdash A & \Longleftrightarrow \exists \ell . \Sigma ; \Gamma \vdash A: \text { Set }_{\ell} & \text { well-formed type } \\
\Sigma ; \Gamma \vdash A=B & \Longleftrightarrow \exists \ell . \Sigma ; \Gamma \vdash A=B: \text { Set }_{\ell} & \text { equal types }
\end{array}
$$

In the inference rules, we make use of substitutions. Substitutions $\sigma, \tau, v$ are partial maps from variable names to terms with a finite domain. If $\operatorname{dom}(\sigma)$ and $\operatorname{dom}(\tau)$ are disjoint, then $\sigma \uplus \tau$ denotes the union of these maps. We write the substitution that maps the variables $x_{1}, \ldots, x_{n}$ to the terms $v_{1}, \ldots, v_{n}$ (and is undefined for all other variables) by $\left[v_{1} / x_{1} ; \ldots ; v_{n} / x_{n}\right]$. In particular, the empty substitution [] is undefined for all variables. If $\Delta=\left(x_{1}: A_{1}\right) \ldots\left(x_{n}: A_{n}\right)$ is a telescope and $\bar{v}=v_{1}, \ldots, v_{n}$ is a list of terms, we may write $[\bar{v} / \Delta]$ for the substitution $[\bar{v} / \hat{\Delta}]$, i.e. $\left[v_{1} / x_{1} ; \ldots ; v_{n} / x_{n}\right]$. In particular, the identity substitution $\mathbb{1}_{\Gamma}=[\hat{\Gamma} / \Gamma]$ maps all variables in $\Gamma$ to themselves. We also use the identity substitution as a weakening substitution, allowing us to forget about all variables that are not in $\Gamma$. If $x \in \operatorname{dom}(\sigma)$, then $\sigma \backslash x$ is defined by removing $x$ from the domain of $\sigma$.

Application of a substitution $\sigma$ to a term $u$ is written as $u \sigma$ and is defined as usual by replacing all (free) variables in $u$ by their values given by $\sigma$, avoiding variable capture via suitable renaming of bound variables. Like function application, this is a partial operation on the syntax; for instance, 
$\Gamma \vdash u: A$ Entails $\vdash \Gamma$ and $\Gamma \vdash A$.

Types.

$$
\begin{aligned}
& \frac{\vdash \Gamma}{\Gamma \vdash \operatorname{Set}_{\ell}: \operatorname{Set}_{\ell+1}} \quad \frac{\Gamma \vdash A: \operatorname{Set}_{\ell} \quad \Gamma(x: A) \vdash B: \operatorname{Set}_{\ell^{\prime}}}{\Gamma \vdash(x: A) \rightarrow B: \operatorname{Set}_{\max \left(\ell, \ell^{\prime}\right)}}
\end{aligned}
$$

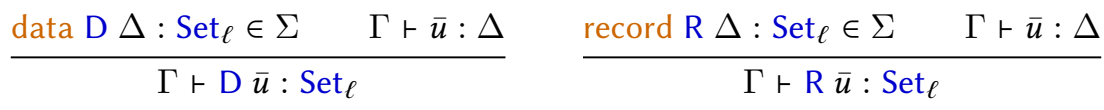

$$
\begin{aligned}
& \frac{\Gamma \vdash A: \operatorname{Set}_{\ell} \quad \Gamma \vdash u: A \quad \Gamma \vdash v: A}{\Gamma \vdash u \equiv_{A} v: \operatorname{Set}_{\ell}}
\end{aligned}
$$

Heads $(h::=x \in \mid f \epsilon)$ and applications $h \bar{e}$.

$$
\frac{\vdash \Gamma \quad x: A \in \Gamma}{\Gamma \vdash x \epsilon: A} \quad \frac{\vdash \Gamma \quad \text { definition } \mathrm{f}: A \in \Sigma}{\Gamma \vdash \mathrm{f} \epsilon: A} \quad \frac{\Gamma \vdash h: A \quad \Gamma \mid h: A \vdash \bar{e}: C}{\Gamma \vdash h \bar{e}: C}
$$

Values.

$$
\frac{\text { constructor c } \Delta_{\mathrm{c}}: \mathrm{D} \Delta \in \Sigma \quad \Gamma \vdash \bar{u}: \Delta \quad \Gamma \vdash \bar{v}: \Delta_{\mathrm{c}}[\bar{u} / \Delta]}{\Gamma \vdash \mathrm{c} \bar{v}: \mathrm{D} \bar{u}} \quad \frac{\Gamma \vdash u: A}{\Gamma \vdash \operatorname{refl}: u \equiv_{A} u}
$$

Conversion.

$$
\frac{\Gamma \vdash u: A \quad \Gamma \vdash A=B}{\Gamma \vdash u: B}
$$

Fig. 2. Typing rules for expressions.

$\Gamma \mid u: A \vdash \bar{e}: C$ If $\Gamma \vdash u: A$ then $\Gamma \vdash C$.

$$
\begin{gathered}
\frac{\Gamma \vdash v: A \quad \Gamma \mid u v: B[v / x] \vdash \bar{e}: C}{\Gamma \mid u: A \vdash \epsilon: A} \quad \frac{\Gamma+u:(x: A) \rightarrow B \vdash v \bar{e}: C}{\text { projection self }: \mathrm{R} \Delta \vdash . \pi: A \in \Sigma \quad \Gamma \mid u \cdot \pi: A[\bar{v} / \Delta, u / \operatorname{self}] \vdash \bar{e}: C} \\
\frac{\Gamma \mid u: \mathrm{R} \bar{v} \vdash . \pi \bar{e}: C}{\Gamma \vdash A=A^{\prime} \quad \Gamma \mid u: A^{\prime} \vdash \bar{e}: C} \\
\Gamma \mid u: A \vdash \bar{e}: C
\end{gathered}
$$

Fig. 3. The typing rules for eliminations.

$(x . \pi)[c / x]$ is undefined as constructors cannot be the head of an elimination. Thus, when a substitution appears in an inference rule, its definedness is an implicit premise of the rule. Also, such pathological cases are ruled out by typing. Well-typed substitutions can always be applied to welltyped terms. Substitution composition $\sigma ; \tau$ shall map the variable $x$ to the term $(x \sigma) \tau$. Note the difference between $\sigma ; \tau$ and $\sigma \uplus \tau$ : the former applies first $\sigma$ and then $\tau$ in sequence, while the 
$\Gamma r_{\ell} \Delta \quad$ Entails $\vdash \Gamma$.

$$
\frac{\vdash \Gamma}{\Gamma \vdash_{\ell} \epsilon} \quad \frac{\Gamma \vdash A: \operatorname{Set}_{\ell^{\prime}} \quad \Gamma(x: A) \vdash_{\ell} \Delta}{\Gamma \vdash_{\ell}(x: A) \Delta} \ell^{\prime} \leq \ell
$$

$\Gamma \vdash \bar{u}: \Delta$ Precondition: $\Gamma \vdash \Delta$.

$$
\overline{\Gamma \vdash \epsilon: \epsilon} \quad \frac{\Gamma \vdash u: A \quad \Gamma \vdash \bar{u}: \Delta[u / x]}{\Gamma \vdash u \bar{u}:(x: A) \Delta}
$$

Fig. 4. The typing rules for telescopes and lists of terms.

latter applies $\sigma$ and $\tau$ in parallel to disjoint parts of the context. Application of a substitution to a pattern $p \sigma$ is defined as $\lceil p\rceil \sigma$.

In addition to substitutions on terms, we also make use of substitutions on patterns called pattern substitutions. A pattern substitution $\rho$ assigns to each variable a pattern. We reuse the same syntax for pattern substitutions as for normal substitutions. Any pattern substitution $\rho$ can be used as a normal substitution $\lceil\rho\rceil$ defined by $x\lceil\rho\rceil=\lceil x \rho\rceil$.

The rules for the typing judgement $\Gamma \vdash t: A$ are listed in Fig. 2. The type formation rules introduce an infinite hierarchy of predicative universes $\operatorname{Set}_{\ell}$ without cumulativity. The formation rules for data and record types make use of the judgment $\Gamma \vdash \bar{u}: \Delta$ to type argument lists, same for the constructor rule, which introduces a data type. Further, refl introduces the equality type. All expressions involved in these rules are fully applied, but this changes when we come to the elimination rules. The types of heads, i.e. variables $x$ or defined constants $f$ are found in the context or signature.

The rules for applying heads $u$ to spines $\bar{e}$, judgement $\Gamma \mid u: A \vdash \bar{e}: C$, are presented in Fig. 3 . For checking arguments, the type of the head is sufficient, and it needs to be a function type. To check projections, we need also the value $u$ of the head that replaces self in the type of the projection. We may need to convert the type of the head to a function or record type to apply these rules, hence, we supply a suitable conversion rule. The result type $C$ of this judgement need not be converted here, it can be converted in the typing judgement for expressions.

Remark 6 (Focused syntax). The reader may have observed that our expressions cover only the non-invertible rules in the sense of focusing [Andreoli 1992], given that we consider data types as multiplicative disjunctions and record types as additive conjunctions: Terms introduce data and eliminate records and functions. The invertible rules, i.e. elimination for data and equality and introduction for function space and records are covered by pattern matching (Sect. 3.4) and, equivalently, case trees (Sect. 4). This matches our intuition that all the information/choice resides with the non-invertible rules, the terms, while the choice-free pattern matching corresponding to the invertible rules only sets the stage for the decisions taken in the terms.

Fig. 4 defines judgement $\Gamma r_{\ell} \Delta$ for telescope formation. The level $\ell$ is an upper bound for the universe levels of the types that comprise the telescope. In particular, if we consider a telescope as a nested $\Sigma$-type, then $\ell$ is an upper bound for the universe that hosts this type. This is important when checking that the level of a data type is sufficiently high for the level of data it contains (Fig. 5). 
$\Sigma+Z$ Snipped $Z$ is well-formed in signature $\Sigma$.

$$
\frac{\Sigma \vdash \Delta}{\Sigma \vdash \text { data D } \Delta: \text { Set } \ell} \quad \frac{\text { data D } \Delta: \operatorname{Set}_{\ell} \in \Sigma \quad \Sigma ; \Delta r_{\ell} \Delta_{\mathrm{c}}}{\Sigma \vdash \text { constructor c } \Delta_{\mathrm{c}}: \mathrm{D} \Delta}
$$

$\frac{\Sigma \vdash \Delta}{\Sigma \vdash \operatorname{record} \mathrm{R} \Delta: \operatorname{Set}_{\ell}} \quad \frac{\text { record R } \Delta: \operatorname{Set}_{\ell} \in \Sigma \quad \Sigma ; \Delta(x: \mathrm{R} \hat{\Delta}) \vdash A: \operatorname{Set}_{\ell^{\prime}}}{\Sigma \vdash \operatorname{projection} x: \mathrm{R} \Delta \vdash . \pi: A} \ell^{\prime} \leq \ell$

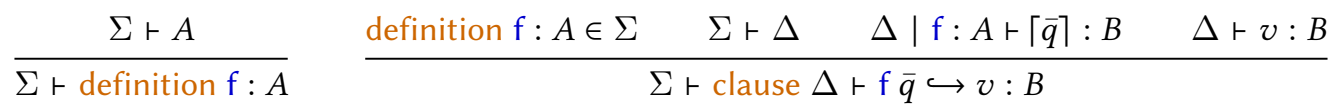

$\Sigma_{0} \subseteq \Sigma$ Signature $\Sigma$ is a valid extension of $\Sigma_{0}$.

$$
\overline{\Sigma_{0} \subseteq \Sigma_{0}} \quad \frac{\Sigma_{0} \subseteq \Sigma \quad \Sigma \vdash Z \quad \Sigma, Z \text { defined }}{\Sigma_{0} \subseteq \Sigma, Z}
$$

Fig. 5. Rules for well-formed signature snippets and extension.

Using the notation $\left(x_{1}, \ldots, x_{n}\right) \sigma=\left(x_{1} \sigma, \ldots, x_{n} \sigma\right)$, substitution typing can be reduced to typing of lists of terms: Suppose $\vdash \Gamma$ and $\vdash \Delta$. We write $\Gamma \vdash \sigma: \Delta$ for $\operatorname{dom}(\sigma)=\Delta$ and $\Gamma \vdash \hat{\Delta} \sigma: \Delta$. Likewise, we write $\Gamma \vdash \sigma=\sigma^{\prime}: \Delta$ for $\Gamma \vdash \hat{\Delta} \sigma=\hat{\Delta} \sigma^{\prime}: \Delta$.

Definitional equality $\Gamma \vdash u=u^{\prime}: A$ is induced by rewriting function applications according to the function clauses. It is the least typed congruence over the axiom:

$$
\frac{\text { clause } \Delta \vdash \mathrm{f} \bar{q} \hookrightarrow v: B \in \Sigma \quad \Gamma \vdash \sigma: \Delta}{\Gamma \vdash \mathrm{f} \bar{q} \sigma=v \sigma: B \sigma}
$$

If $\mathrm{f} \bar{q} \hookrightarrow v$ is a defining clause of function $\mathrm{f}$, then each instance arising from a well-typed substitution $\sigma$ is a valid equation. The full list of congruence and equivalence rules is given in the long version of this paper, together with congruence rules for applications and lists of terms. As usual in dependent type theory, definitional equality on types $\Gamma \vdash A=B: \operatorname{Set}_{\ell}$ is used for type conversion.

\subsection{Signature Well-formedness}

A signature $\Sigma$ extends $\Sigma_{0}$ if we can go from $\Sigma_{0}$ to $\Sigma$ by adding valid snippets $Z$, i.e. new datatypes, record types, and defined constants, but new constructors/projections/clauses only for not yet completed definitions in $\Sigma$. A signature $\Sigma$ is well-formed if it is a valid extension of the empty signature $\epsilon$. Formally, we define signature extension $\Sigma_{0} \subseteq \Sigma$ via snippet typing $\Sigma \vdash Z$ by the rules in Fig. 5, and signature well-formedness $+\Sigma$ as $\epsilon \subseteq \Sigma$. Recall that the rules for extending the signature with a constructor (resp. projection or clause) can only be used when the corresponding data type (resp. record type or definition) is the last thing in the signature, by definition of extending the signature with a snippet $\Sigma, Z$. When adding a constructor or projection, it is ensured that the stored data is not too big in terms of universe level $\ell$; this preserves predicativity. However, the parameters $\Delta$ of a data or record type of level $\ell$ can be big, they may exceed $\ell$.

All typing and equality judgements are monotone in the signature, thus, remain valid under signature extensions. 
$\Sigma \vdash u \searrow w \quad(\Sigma$ fixed, dropped from rules.)

$\overline{w \searrow w} \frac{\text { clause } \Delta \vdash \mathrm{f} \bar{q} \hookrightarrow v: A \in \Sigma \quad[\bar{e} / \bar{q}] \searrow \sigma \quad v \sigma \searrow w}{\mathrm{f} \bar{e} \searrow w}$

Fig. 6. Rules for weak head normalization.

$\Sigma \vdash[v / p] \searrow \sigma_{\perp} \quad(\Sigma$ fixed, dropped from rules.)

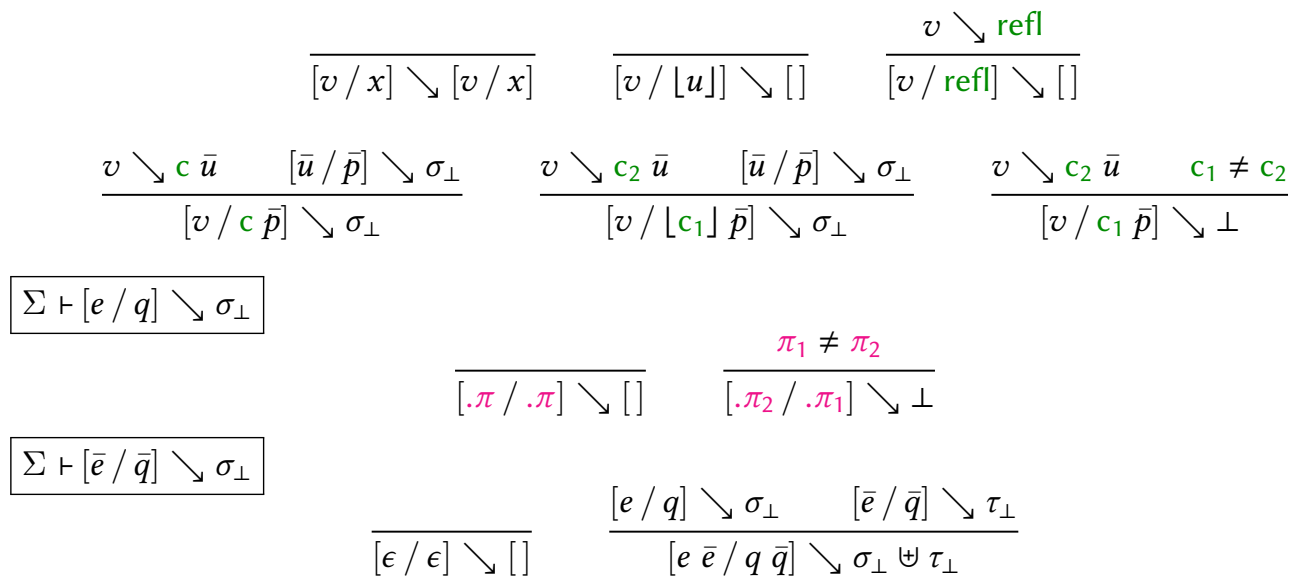

Fig. 7. Rules for the pattern matching and mismatching algorithm.

Lemma 7 (Signature extension preserves inferences). If $\Sigma ; \Gamma \vdash u: A$ and $\Sigma \subseteq \Sigma^{\prime}$ then also $\Sigma^{\prime} ; \Gamma \vdash u: A$ (and likewise for other judgements).

Remark 8 (Coverage). The rules for extending a signature with a function definition given by a list of clauses are not strong enough to guarantee the usual properties of a language such as type preservation and progress. For example, we could define a function with no clauses at all (violating progress), or we could add a clause where all patterns are forced patterns (violating type preservation). We prove type preservation and progress only for functions that correspond to a well-typed case tree as defined in Sect. 4.

\subsection{Pattern Matching and Evaluation Rules}

Evaluation to weak head normal form $\Sigma \vdash u \searrow w$ is defined inductively in Fig. 6. Since our language does not contain syntax for lambda abstraction, there is no rule for $\beta$-reduction. Almost all terms are their own weak head normal form; the only exception are applications $\mathrm{f} \bar{e}$.

Evaluation is mutually defined with matching against (co)patterns $\sum \vdash[\bar{e} / \bar{q}] \searrow \sigma_{\perp}$ (Fig. 7). Herein, $\sigma_{\perp}$ is either a substitution $\sigma$ with $\operatorname{dom}(\sigma)=\mathrm{PV}(\bar{q})$ or the error value $\perp$ for mismatch. Join of lifted substitutions $\sigma_{\perp} \uplus \tau_{\perp}$ is $\perp$ if one of the operands is $\perp$, otherwise the join $\sigma \uplus \tau$.

A pattern variable $x$ matches any term $v$, producing singleton substitution $[v / x]$. Likewise for a forced pattern $\lfloor u\rfloor$, but it does not bind any pattern variables. Projections . $\pi$ only match themselves, and so do constructors c $\bar{p}$, but they require evaluation $v \searrow \mathrm{c} \bar{u}$ of the scrutinee $v$ and subsequent 
successful matching $[\bar{u} / \bar{p}] \searrow \sigma$ of the arguments. For forced constructors $\left\lfloor c_{1}\right\rfloor \bar{p}$, the constructor equality test is skipped, as it is ensured by typing. Constructor $\left(c_{1} \neq c_{2}\right)$ and projection $\left(. \pi_{1} \neq . \pi_{2}\right)$ mismatches produce $\perp$. We do not need to match against the absurd pattern; user clauses with absurd matches are never added to the signature. Recall that absurd patterns are not contained in clauses of the signature, thus, we need not consider them in the matching algorithm. Evaluating a function that eliminates absurdity will be stuck for lack of matching clauses.

A priori, matching can get stuck, if none of the rules apply. In particular, this happens when we try to evaluate an underapplied function or an open term, i.e. a term with free variables. For the purpose of the evaluation judgement, we would not need to track definite mismatch $(\perp)$ separately from getting stuck. However, for the first-match semantics [Augustsson 1985] we do: There, a function should reduce with the first clause that matches while all previous clauses produce a mismatch. If matching a clause is stuck, we must not try the next one.

The first-match semantics is also the reason why either $\Sigma \vdash[e / q] \searrow \perp$ or $\Sigma \vdash[\bar{e} / \bar{q}] \searrow \perp$ alone is not sufficient to derive $\Sigma \vdash[e \bar{e} / q \bar{q}] \searrow \perp$, i.e. mismatch does not dominate stuckness, nor does it short-cut matching. Suppose a function and defined by the clauses true true $\hookrightarrow$ true and $x y \hookrightarrow$ false. If mismatch dominated stuckness, then both open terms and false $y$ and and $x$ false would reduce to false. However, there is no case tree that accomplishes this. We have to split on the first or the second variable; either way, one of the two open terms will be stuck. We cannot even decree left-to-right splitting: see Example 5 for a definition that is impossible to elaborate to a case tree using a left-to-right splitting order. Thus, we require our pattern match semantics to be faithful with any possible elaboration of clauses into case trees. ${ }^{8}$

\subsection{Other Language Features}

In comparison to dependently typed programming languages like Agda and Idris, our core language seems rather reduced. In the following, we discuss how some popular features could be translated to our core language.

Lambda abstractions and $\eta$-equality: A lambda abstraction $\lambda x . t$ in context $\Gamma$ can be lifted to the top-level and encoded as auxiliary function $\mathrm{f} \hat{\Gamma} x \hookrightarrow t$. We obtain extensionality $(\eta)$ by adding the following rule to definitional equality:

$$
\frac{\Gamma \vdash t_{1}:(x: A) \rightarrow B \quad \Gamma \vdash t_{2}:(x: A) \rightarrow B \quad \Gamma(x: A) \vdash t_{1} x=t_{2} x: B}{\Gamma \vdash t_{1}=t_{2}:(x: A) \rightarrow B} x \notin \operatorname{dom}(\Gamma)
$$

Record expressions: Likewise, a record value record $\{\bar{\pi}=\bar{v}\}$ in $\Gamma$ can be turned into an auxiliary definition by copattern matching with clauses $\left(\mathrm{f} \hat{\Gamma} \cdot \pi_{\mathrm{i}} \hookrightarrow v_{i}\right)_{i}$. We could add an $\eta$-law that considers two values of record type $\mathrm{R}$ definitionally equal if they are so under each projection of R. However, to maintain decidability of definitional equality, this should only applied to non-recursive records, as recursive records model coinductive types which do not admit $\eta$.

Indexed datatypes can be defined as regular (parameterized) datatypes with extra arguments to each constructor containing equality proofs for the indices. For example, Vec $A n$ can be defined as follows:

$$
\begin{aligned}
& \text { data } \operatorname{Vec}\left(A: \operatorname{Set}_{\ell}\right)(n: \mathbb{N}): \text { Set }_{\ell} \text { where } \\
& \text { nil }: n \equiv_{\mathbb{N}} \text { zero } \rightarrow \operatorname{Vec} A n \\
& \text { cons }:(m: \mathbb{N})(x: A)(x s: \operatorname{Vec} A m) \rightarrow n \equiv_{\mathbb{N}} \text { suc } m \rightarrow \operatorname{Vec} A n
\end{aligned}
$$

\footnotetext{
${ }^{8}$ In a sense, this is opposite to lazy pattern matching [Maranget 1992], which aims to find the right clause with the least amount of matching.
} 
Indexed record types can be defined analogously to indexed datatypes. For example, Vec $A n$ can also be defined as a record type:

$$
\begin{aligned}
& \text { record Vec }\left(A: \text { Set }_{\ell}\right)(n: \mathbb{N}): \text { Set } \ell \text { where } \\
& \quad \text { head }:(m: \mathbb{N}) \rightarrow n \equiv_{\mathbb{N}} \text { suc } m \rightarrow A \\
& \text { tail } \quad:(m: \mathbb{N}) \rightarrow n \equiv_{\mathbb{N}} \text { suc } m \rightarrow \operatorname{Vec} A m
\end{aligned}
$$

The 'constructors' nil and cons are then defined by

$$
\begin{array}{ll}
\text { nil : Vec } A \text { zero } & \text { cons }:(n: \mathbb{N})(x: A)(x s: \text { Vec } A n) \rightarrow \operatorname{Vec} A(\text { suc } n) \\
\text { nil .head } m \emptyset=\text { impossible } & \text { cons } n \times x \text { s . head }\lfloor n\rfloor \text { refl }=x \\
\text { nil .tail } m \emptyset=\text { impossible } & \text { cons } n \times x \text { s .tail }\lfloor n\rfloor \text { refl }=x s
\end{array}
$$

Mutual recursion can be simulated by nested recursion as long as we do not define checks for positivity and termination.

Wildcard patterns can be written as variable patterns with a fresh name. Note that an unused variable may stand for either a wildcard or a forced pattern. In the latter case our algorithm treats it as a let-bound variable in the right-hand side of the clause.

Record patterns would make sense for inductive records with $\eta$. Without changes to the core language, we can represent them by first turning deep matching into shallow matching, along the lines of Setzer et al. [2014], and then turn record matches on the left-hand side into projection applications on the right-hand side.

This concludes the presentation of our core language.

\section{CASE TREES}

From a user perspective it is nice to be able to define a function by a list of clauses, but for a core language this representation of functions leaves much to be desired: it is hard to see whether a set of clauses is covering all cases [Coquand 1992], and evaluating the clauses directly can be slow for deeply nested patterns [Cardelli 1984]. Recall that for type-checking dependent types, we need to decide equality of open terms which requires computing weak head normal forms efficiently.

Thus, instead of using clauses, we represent functions by a case tree in our core language. In this section, we give a concrete syntax for case trees and give typing and evaluation rules for them. We also prove that a function defined by a case tree enjoys good properties such as type preservation and coverage.

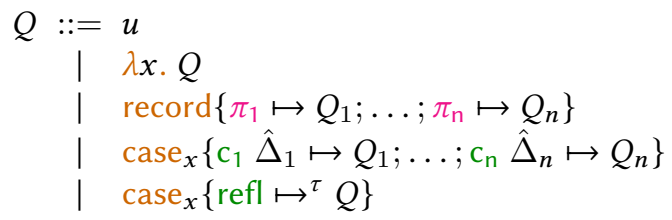

branch body (splitting done) bringing next argument into scope as $x$ splitting result by possible projections $(n \geq 0)$

splitting on data $x(n \geq 0)$ matching on equality proof $x$

Note that empty case and empty record are allowed, to cover the empty data type and the unit type, i.e. the record without fields.

Remark 9 (Focusing). Case trees allow us to introduce functions and records, and eliminate data. In the sense of focusing, this corresponds to the invertible rules for implication, additive conjunction, and multiplicative disjunction. (See typing rules in Fig. 8.)

\subsection{Case Tree Typing}

A case tree $Q$ for a defined constant $\mathrm{f}: A$ is well-typed in environment $\Sigma$ if $\Sigma \vdash \mathrm{f}:=Q: A \leadsto \Sigma^{\prime}$. In this judgement, $\Sigma$ is the signature in which case tree $Q$ for function $\mathrm{f}: A$ is well-typed, and $\Sigma^{\prime}$ 
$\Sigma ; \Gamma \vdash \mathrm{f} \bar{q}:=Q: C \sim \Sigma^{\prime}$ Presupposes: $\Sigma ; \Gamma \vdash \mathrm{f}\lceil\bar{q}\rceil: C$ and $\operatorname{dom}(\Gamma)=\operatorname{PV}(\bar{q})$.

Checks case tree $Q$ and outputs an extension $\Sigma^{\prime}$ of $\Sigma$ by the clauses represented by "f $\bar{q} \hookrightarrow Q$ ".

$$
\begin{aligned}
& \frac{\Sigma ; \Gamma \vdash v: C}{\Sigma ; \Gamma \vdash f \bar{q}:=v: C \leadsto \Sigma,(\text { clause } \Gamma \vdash \mathrm{f} \bar{q} \hookrightarrow v: C)} \text { CTDone } \\
& \frac{\Sigma ; \Gamma \vdash C=(x: A) \rightarrow B: \operatorname{Set}_{\ell} \quad \Sigma ; \Gamma(x: A) \vdash \mathrm{f} \bar{q} x:=Q: B \leadsto \Sigma^{\prime}}{\Sigma ; \Gamma \vdash \mathrm{f} \bar{q}:=\lambda x \cdot Q: C \leadsto \Sigma^{\prime}} \text { CтIntro } \\
& \Sigma_{0} ; \Gamma \vdash C=\mathrm{R} \bar{v}: \text { Set } \ell \quad \text { record self: } \mathrm{R} \Delta: \text { Set }_{\ell} \text { where } \overline{\pi_{\mathrm{i}}: A_{i}} \in \Sigma_{0} \\
& \frac{\sigma=[\bar{v} / \Delta, \mathrm{f}\lceil\bar{q}\rceil / \text { self }] \quad\left(\Sigma_{i-1} ; \Gamma \vdash \mathrm{f} \bar{q} \cdot \pi_{\mathrm{i}}:=Q_{i}: A_{i} \sigma \leadsto \Sigma_{i}\right)_{i=1 \ldots n}}{\Sigma_{0} ; \Gamma \vdash \mathrm{f} \bar{q}:=\operatorname{record}\left\{\pi_{1} \mapsto Q_{1} ; \ldots ; \pi_{\mathrm{n}} \mapsto Q_{n}\right\}: C \leadsto \Sigma_{n}} \text { CтCosplit } \\
& \Sigma_{0} ; \Gamma_{1} \vdash A=\mathrm{D} \bar{v}: \text { Set }_{\ell} \quad \text { data } \mathrm{D} \Delta: \text { Set } \ell \text { where } \overline{\mathrm{c}_{\mathrm{i}} \Delta_{i}} \in \Sigma_{0} \\
& \left(\Delta_{i}^{\prime}=\Delta_{i}[\overline{\mathcal{v}} / \Delta]\right)_{i=1 \ldots n} \quad\left(\rho_{i}=\mathbb{1}_{\Gamma_{1}} \uplus\left[\mathrm{c}_{\mathrm{i}} \hat{\Delta}_{i}^{\prime} / x\right]\right)_{i=1 \ldots n} \\
& \frac{\left(\rho_{i}^{\prime}=\rho_{i} \uplus \mathbb{1}_{\Gamma_{2}}\right)_{i=1 \ldots n} \quad\left(\Sigma_{i-1} ; \Gamma_{1} \Delta_{i}^{\prime}\left(\Gamma_{2} \rho_{i}\right) \vdash \mathrm{f} \bar{q} \rho_{i}^{\prime}:=Q_{i}: C \rho_{i}^{\prime} \leadsto \Sigma_{i}\right)_{i=1 \ldots n}}{\Sigma_{0} ; \Gamma_{1}(x: A) \Gamma_{2} \vdash \mathrm{f} \bar{q}:=\operatorname{case}_{x}\left\{\mathrm{c}_{1} \hat{\Delta}_{1}^{\prime} \mapsto Q_{1} ; \ldots ; \mathrm{c}_{\mathrm{n}} \hat{\Delta}_{n}^{\prime} \mapsto Q_{n}\right\}: C \leadsto \Sigma_{n}} \text { CтSplitCon } \\
& \Sigma ; \Gamma_{1} \vdash A=\left(u \equiv_{B} v\right): \text { Set }_{\ell} \quad \Sigma ; \Gamma_{1} \vdash_{x} u=? v: B \Rightarrow \operatorname{yes}\left(\Gamma_{1}^{\prime}, \rho, \tau\right) \\
& \frac{\rho^{\prime}=\rho \uplus \mathbb{1}_{\Gamma_{2}} \quad \tau^{\prime}=\tau \uplus \mathbb{1}_{\Gamma_{2}} \quad \Sigma ; \Gamma_{1}^{\prime}\left(\Gamma_{2} \rho\right) \vdash \mathrm{f} \bar{q} \rho^{\prime}:=Q: C \rho^{\prime} \leadsto \Sigma^{\prime}}{\Sigma ; \Gamma_{1}(x: A) \Gamma_{2} \vdash \mathrm{f} \bar{q}:=\operatorname{case}_{x}\left\{\operatorname{refl} \mapsto \tau^{\tau^{\prime}} Q\right\}: C \leadsto \Sigma^{\prime}} \text { CTSplitEQ } \\
& \frac{\Sigma ; \Gamma_{1} \vdash A=\left(u \equiv_{B} v\right): \operatorname{Set}_{\ell} \quad \Sigma ; \Gamma_{1} \vdash_{x} u=? v: B \Rightarrow \text { NO }}{\Sigma ; \Gamma_{1}(x: A) \Gamma_{2} \vdash f \bar{q}:=\operatorname{case}_{x}\{\}: C \sim \Sigma} \text { CTSPlitAbsurdEQ }
\end{aligned}
$$

Fig. 8. The typing rules for case trees.

is the output signature which is $\Sigma$ extended with the function clauses corresponding to case tree $Q$. Note that the absence of a local context $\Gamma$ in this proposition implies that we only use case trees for top-level definitions. ${ }^{9}$

Case tree typing is established by the generalized judgement $\Sigma ; \Gamma \vdash \mathrm{f} \bar{q}:=Q: A \leadsto \Sigma^{\prime}$ (Fig. 8) that considers a case tree $Q$ for the instance $f \bar{q}$ of the function in a context $\Gamma$ of the pattern variables of $\bar{q}$. We have the following rules for $\Sigma ; \Gamma \vdash \mathrm{f} \bar{q}:=Q: A \leadsto \Sigma^{\prime}$ :

CTDone A leaf of a case tree consists of a right-hand side $v$ which needs to be of the same type $C$ of the corresponding left-hand side $f \bar{q}$ and may only refer to the pattern variables $\Gamma$ of $\bar{q}$. If this is the case, the clause $\mathrm{f} \bar{q} \hookrightarrow v$ is added to the signature.

CTINTro If the left-hand side $\mathrm{f} \bar{q}$ is of function type $(x: A) \rightarrow B$ we can extend it by variable pattern $x$. The corresponding case tree is function introduction $\lambda x . Q$.

CTCosplit If the left-hand side is of record type $\mathrm{R} \bar{v}$ with projections $\pi_{\mathrm{i}}$, we can do result splitting and extend it by copattern . $\pi_{\mathrm{i}}$ for all $i$. We have record $\left\{\pi_{1} \mapsto Q_{1} ; \ldots ; \pi_{\mathrm{n}} \mapsto Q_{n}\right\}$ (where $n \geq 0$ ) as the corresponding case tree, and we check each sub tree $Q_{i}$ for left-hand side $\mathrm{f} \bar{q} \cdot \pi_{\mathrm{i}}$ in the signature $\Sigma_{i-1}$ which includes the clauses for the branches $j<i$. Note that

\footnotetext{
${ }^{9}$ It would also be possible to embed case trees into our language as terms instead, as is the case in many other languages. We refrain from doing so in this paper for the sake of simplicity.
} 
these previous clauses may be needed to check the current case, since we have dependent records (Example 2).

CTSPlitCon If left-hand side $\mathrm{f} \bar{q}$ contains a variable $x$ of data type $\mathrm{D} \bar{v}$, we can split on $x$ and consider all alternatives $\mathrm{c}_{\mathrm{i}}$, generating the case tree $\operatorname{case}_{x}\left\{\mathrm{c}_{1} \hat{\Delta}_{1}^{\prime} \mapsto Q_{1} ; \ldots ; \mathrm{c}_{\mathrm{n}} \hat{\Delta}_{n}^{\prime} \mapsto Q_{n}\right\}$. The branch $Q_{i}$ is checked for a refined left-hand side where $x$ has been substituted by c $\hat{\Delta}_{i}^{\prime}$ in a context where $x$ has been replaced by the new pattern variables $\Delta_{i}^{\prime}$. Note also the threading of signatures as in rule СтCоspLit.

The rules CTSPLitEq and CTSplitAbsurdEQ are explained in the next section.

\subsection{Unification: Splitting on the Identity Type}

To split on an equality proof $x: u \equiv_{B} v$ we try to unify $u$ and $v$. We may either find a most general unifier (m.g.u.); then we can build a case tree case $_{x}\{$ refl $\mapsto \cdot\}$ (rule CTSplitEQ). We may find a disunifier and build the case tree case $_{x}\{\}$ (rule CTSplitAbsurdEQ). Finally, there might be neither a m.g.u. nor a disunifier, e.g. for equality $y+z \equiv_{\mathbb{N}} y^{\prime}+z^{\prime}$; then type-checking fails.

In fact, in our setting we need a refinement of m.g.u.s we call strong unifiers. We recall the definitions of a strong unifier and a disunifier from Cockx et al. [2016], here translated to the language of this paper and specialized to the case of a single equation:

Definition 10 (Strong unifier). Let $\Gamma$ be a well-formed context and $u$ and $v$ be terms such that $\Gamma \vdash u, v:$ A. A strong unifier $\left(\Gamma^{\prime}, \sigma, \tau\right)$ of $u$ and $v$ consists of a context $\Gamma^{\prime}$ and substitutions $\Gamma^{\prime} \vdash \sigma$ : $\Gamma\left(x: u \equiv_{A} v\right)$ and $\Gamma\left(x: u \equiv_{A} v\right) \vdash \tau: \Gamma^{\prime}$ such that:

(1) $\Gamma^{\prime} \vdash x \sigma=\operatorname{refl}: u \sigma \equiv_{A \sigma} v \sigma \quad$ (this implies the definitional equality $\Gamma^{\prime} \vdash u \sigma=v \sigma: A \sigma$ )

(2) $\Gamma^{\prime} \vdash \tau ; \sigma=\mathbb{1}_{\Gamma^{\prime}}: \Gamma^{\prime}$

(3) For any context $\Gamma_{0}$ and substitution $\sigma_{0}$ such that $\Gamma_{0} \vdash \sigma_{0}: \Gamma\left(x: u \equiv_{A} v\right)$ and $\Gamma_{0} \vdash x \sigma_{0}=$ refl : $u \sigma_{0} \equiv_{A \sigma_{0}} v \sigma_{0}$, we have $\Gamma_{0} \vdash \sigma ; \tau ; \sigma_{0}=\sigma_{0}: \Gamma\left(x: u \equiv_{A} v\right)$.

Definition 11 (Disunifier). Let $\Gamma$ be a well-formed context and $\Gamma \vdash u, v:$ A. A disunifier of $u$ and $v$ is a function $\Gamma \vdash f:\left(u \equiv_{A} v\right) \rightarrow \perp$ where $\perp$ is the empty type.

Since we use the substitution $\sigma$ for the construction of the left-hand side of clauses, we require unification to output not just a substitution but a pattern substitution $\rho$. The only properly matching pattern in $\rho$ is $x \rho=$ refl; all the other patterns $y \rho$ are either a forced pattern $\lfloor t\rfloor$ (if unification instantiates $y$ with $t$ ) or the variable $y$ itself (if unification leaves $y$ untouched).

We thus assume we have access to a proof relevant unification algorithm specified by the following judgements:

- $\Sigma ; \Gamma \vdash_{x} u={ }^{?} v: A \Rightarrow \operatorname{yes}\left(\Gamma^{\prime}, \rho, \tau\right)$ ensures that $x \rho=\operatorname{refl}$ and the triple $\left(\Gamma^{\prime},\lceil\rho\rceil, \tau\right)$ is a strong unifier. Additionally, $\Gamma^{\prime} \subseteq \Gamma, y \tau=y$ and $y \rho=y$ for all $y \in \Gamma^{\prime}$, and $y \rho$ is a forced pattern for all variables $y \in \Gamma \backslash \Gamma^{\prime}$.

- $\Sigma ; \Gamma \vdash_{x} u=? v: A \Rightarrow$ No ensures that there exists a disunifier of $u$ and $v$.

Remark 12. During the unification of $u$ with $v$, each step either instantiates one variable from $\Gamma$ (e.g. the solution step) or leaves it untouched (e.g. the injectivity step). We thus have the invariant that the variables in $\Gamma^{\prime}$ form a subset of the variables in $\Gamma$. In effect, the substitution $\tau$ makes the variables instantiated by unification go 'out of scope' after a match on refl. This property ceases to hold in a language with $\eta$-equality for record types and unification rules for $\eta$-expanding a variable such as the ones given by Cockx et al. [2016]. In particular, $\tau$ may contain not only variables but also projections applied to those variables. 
$\Sigma \vdash Q \sigma \bar{e} \longrightarrow v(\Sigma$ dropped from rules, used only for weak head normalization $\Sigma \vdash t \searrow w$.

$\overline{v \sigma \bar{e} \longrightarrow v \sigma \bar{e}}$

$$
\begin{gathered}
\frac{Q(\sigma \uplus[u / x]) \bar{e} \longrightarrow v}{(\lambda x . Q) \sigma u \bar{e} \longrightarrow v} \quad \frac{Q_{i} \sigma \bar{e} \longrightarrow v}{\left(\operatorname{record}\left\{\pi_{1} \mapsto Q_{1} ; \ldots ; \pi_{\mathrm{n}} \mapsto Q_{n}\right\}\right) \sigma . \pi_{\mathrm{i}} \bar{e} \longrightarrow v} \\
\frac{x \sigma \searrow \mathrm{c}_{i} \bar{u} \quad Q_{i}\left(\sigma \backslash x \uplus\left[\bar{u} / \hat{\Delta}_{i}\right]\right) \bar{e} \longrightarrow v}{\left(\operatorname{case}_{x}\left\{\mathrm{c}_{1} \hat{\Delta}_{1} \mapsto Q_{1} ; \ldots ; \mathrm{c}_{\mathrm{n}} \hat{\Delta}_{n} \mapsto Q_{n}\right\}\right) \sigma \bar{e} \longrightarrow v} \quad \frac{x \sigma \searrow \operatorname{refl} \quad Q(\tau ; \sigma) \bar{e} \longrightarrow v}{\left(\operatorname{case}_{x}\left\{\operatorname{refl}^{\tau} Q\right\}\right) \sigma \bar{e} \longrightarrow v}
\end{gathered}
$$

Fig. 9. Evaluation of case trees.

\subsection{Operational Semantics}

If a function $\mathrm{f}$ is defined by a case tree $Q$, then we can compute the application of $\mathrm{f}$ to eliminations $\bar{e}$ via the judgement $\Sigma \vdash Q \sigma \bar{e} \longrightarrow v$ (Fig. 9) with $\sigma=$ []. The substitution $\sigma$ acts as an accumulator, collecting the values for each of the variables introduced by a $\lambda$ or by the constructor arguments in a case $_{x}\{\ldots\}$. In particular, when evaluating a case tree of the form case $x\left\{\right.$ refl $\left.\mapsto^{\tau} Q\right\}$, the substitution $\tau$ is used to remove any bindings in $\sigma$ that correspond to forced patterns.

\subsection{Properties}

If a function $\mathrm{f}$ is defined by a well-typed case tree, then it enjoys certain good properties such as type preservation and coverage. The goal of this section is to state these properties, the corresponding proofs can be found in the long version of this paper. First, we need some basic lemmata.

Lemma 13 (Well-typed case trees preserve signature well-formedness). Let $\vdash \Sigma$ be a well-formed signature with definition $\mathrm{f}: A$ where $\overline{c l s}^{\oplus}$ the last declaration in $\Sigma$ and let $Q$ be a case tree such that $\Sigma ; \Gamma \vdash \mathrm{f} \bar{q}:=Q: C \leadsto \Sigma^{\prime}$ where $\Sigma \vdash \Gamma$ and $\Sigma ; \Gamma \mid \mathrm{f}: A \vdash\lceil\bar{q}\rceil: C$. Then $\Sigma^{\prime}$ is also well-formed.

Proof. By induction on $\Sigma ; \Gamma \vdash \mathrm{f} \bar{q}:=Q: C \leadsto \Sigma^{\prime}$.

The following lemma implies that once the typechecker has completed checking a definition, we can replace the clauses of that definition by the case tree. This gives us more efficient evaluation of the function and guarantees that evaluation is deterministic.

Lemma 14 (Simulation lemma). Consider a case tree $Q$ such that $\Sigma_{0} ; \Gamma \vdash \mathrm{f} \bar{q}:=Q: C \leadsto \Sigma$, let $\sigma$ be a substitution with domain the pattern variables of $\bar{q}$, and let $\bar{e}$ be some eliminations. If $\Sigma \vdash Q \sigma \bar{e} \longrightarrow$ $t$ then there is some pattern substitution $\rho$ and copatterns $\bar{q}^{\prime}$ such that clause $\Delta \vdash \mathrm{f} \bar{q} \rho \bar{q}^{\prime} \hookrightarrow v: A$ is in $\Sigma \backslash \Sigma_{0}$ and $t=v \theta \bar{e}_{2}$ where $\Sigma \vdash\left[\bar{q} \sigma \bar{e}_{1} / \bar{q} \rho \bar{q}^{\prime}\right] \searrow \theta$ and $\bar{e}=\bar{e}_{1} \bar{e}_{2}$.

Conversely, any clause in $\Sigma \backslash \Sigma_{0}$ is of the form clause $\Delta+\mathrm{f} \bar{q} \rho \bar{q}^{\prime} \hookrightarrow v: A$, and for any $\sigma$ and $\bar{e}_{1}$ and $\bar{e}_{2}$ such that $\Sigma \vdash\left[\bar{q} \sigma \bar{e}_{1} / \bar{q} \rho \bar{q}^{\prime}\right] \searrow \theta$ we have $\Sigma \vdash Q \sigma \bar{e}_{1} \bar{e}_{2} \longrightarrow v \theta \bar{e}_{2}$.

Proof. By induction on $Q$.

Before adding a clause $\mathrm{f} \bar{q} \hookrightarrow v$ to the signature, we have to make sure that the copatterns $\bar{q}$ only use forced patterns in places where it is justified: otherwise we might have $\Sigma \vdash[\bar{e} / \bar{q}] \searrow \sigma$ but $\lceil\bar{q}\rceil \sigma \neq \bar{e}$. This is captured in the notion of a respectful pattern [Goguen et al. 2006]. Here we generalize their definition to the case where we do not yet know that all reductions in the signature are necessarily type-preserving. 
Definition 15. A signature $\Sigma$ is respectful for $\Sigma \vdash u \searrow w$ if $\Sigma ; \Gamma \vdash u: A$ implies $\Sigma ; \Gamma \vdash u=w: A$. A signature $\Sigma$ is respectful if it is respectful for all derivations of $\Sigma \vdash u \searrow w$.

In particular, this means $\Sigma ; \Gamma \vdash w: A$, so evaluation with signature $\Sigma$ is type preserving. It is immediately clear that the empty signature is respectful, since it does not contain any clauses.

Definition 16 (Respectful copatterns). Let $\bar{q}$ be a list of copatterns such that $\Sigma ; \Delta \mid u: A \vdash\lceil\bar{q}\rceil: C$ where $u$ and $A$ are closed (i.e. do not depend on $\Delta$ ). We call $\bar{q}$ respectful in signature $\Sigma$ if the following holds: for any signature extension $\Sigma \subseteq \Sigma^{\prime}$ and any eliminations $\Sigma^{\prime} ; \Gamma \mid u: A \vdash \bar{e}: C$ such that $\Sigma^{\prime} \vdash[\bar{e} / \bar{q}] \searrow \sigma$ and $\Sigma^{\prime}$ is respectful for any $\Sigma^{\prime} \vdash s \searrow t$ used in the derivation of $\Sigma^{\prime} \vdash[\bar{e} / \bar{q}] \searrow \sigma$, we have $\Sigma^{\prime} ; \Gamma \mid u: A \vdash \bar{q} \sigma=\bar{e}: C$.

Being respectful is stable under signature extension by definition: if $\bar{q}$ is respectful in $\Sigma$ and $\Sigma \subseteq \Sigma^{\prime}$, then $\bar{q}$ is also respectful in $\Sigma^{\prime}$.

Lemma 17 (Signatures with respectful clauses are respectful). If $\Sigma$ is a well-formed signature such that all clauses in $\Sigma$ have respectful copatterns in $\Sigma$, then $\Sigma$ is respectful.

Proof. By induction on the derivation of $\Sigma \vdash u \searrow v$.

Lemma 18 (Well-typed case trees have respectful clauses). Consider a respectful signature $\Sigma_{0}$ and a case tree $Q$ such that $\Sigma_{0} ; \Gamma \vdash \mathrm{f} \bar{q}:=Q: C \leadsto \Sigma$ and $\bar{q}$ is respectful in $\Sigma_{0}$. Then all clauses in $\Sigma \backslash \Sigma_{0}$ have respectful patterns in $\Sigma$.

Proof. By induction on the derivation of $\Sigma_{0} ; \Gamma \vdash \mathrm{f} \bar{q}:=Q: C \leadsto \Sigma$.

Theorem 19 (Type preservation). If all functions in a signature $\Sigma$ are given by well-typed case trees, then $\Sigma$ is respectful.

Proof. This is a direct consequence of the previous two lemmata.

Definition 20. A term $u$ is normalizing in a signature $\Sigma$ if $\Sigma \vdash u \searrow w$, and additionally, if $w=\mathrm{c} \bar{v}$ then all $\bar{v}$ are also normalizing.

An elimination $e$ is normalizing if it is either a projection.$\pi$ or a normalizing term $u$. A substitution $\sigma$ is normalizing if $x \sigma$ is normalizing for all variables $x$ in $\operatorname{dom}(\sigma)$.

The definition of a normalizing term (and the proof of the following lemma) would be somewhat more complicated for a language with eta-equality for record types, such as the one used by Cockx et al. [2016]. In particular, all projections of a normalizing expression of record type should also be normalizing.

Lemma 21. Suppose $\Sigma ; \Gamma \vdash_{x} u={ }^{?} v: A \Rightarrow \operatorname{YES}\left(\Gamma^{\prime}, \rho, \tau\right)$ and $\Sigma \vdash \sigma_{0}: \Gamma$ such that $\Sigma \vdash u \sigma_{0}=v \sigma_{0}$ : $A \sigma_{0}$. If $\sigma_{0}$ is normalizing, then so is $\tau ; \sigma_{0}$.

Proof. By construction, $y \tau=y$ for each variable $y$ in $\Gamma^{\prime}$ (see Remark 12), so it follows trivially that $\tau ; \sigma_{0}$ is normalizing.

Theorem 22 (Coverage). Let $Q$ be a case tree such that $\Sigma_{0} ; \Gamma \vdash \mathrm{f} \bar{q}:=Q: C \leadsto \Sigma$. Let further $\Sigma \vdash \sigma_{0}: \Gamma$ be a (closed) substitution and $\Sigma \mid \mathrm{f} \bar{q} \sigma_{0}: C \sigma_{0} \vdash \bar{e}: B$ be (closed) eliminations such that $\sigma_{0}$ and $\bar{e}$ are normalizing in $\Sigma$ and $B$ is not definitionally equal to a function type or a record type. Then $\Sigma \vdash Q \sigma_{0} \bar{e} \longrightarrow v$ for some $v$.

Proof. By induction on the case tree $Q$.

In particular, this theorem tells us that if $\Sigma_{0} \vdash \mathrm{f}:=Q: C \leadsto \Sigma$ and the eliminations $\Sigma \mid \mathrm{f}$ : $C \vdash \bar{e}: A$ are normalizing, then $\Sigma \vdash Q[] \bar{e} \longrightarrow v$. Thus evaluation of a function defined by a well-typed case tree applied to closed arguments can never get stuck. 
$\Sigma \vdash \operatorname{decl} \leadsto \Sigma^{\prime}$ Presupposes: $\vdash \Sigma$. Entails: $\vdash \Sigma^{\prime}$.

$$
\begin{gathered}
\frac{\Sigma \vdash \Delta \quad\left(\Sigma, \text { data D } \Delta: \text { Set }_{\ell}\right) \mid \mathrm{D} \Delta: \text { Set }_{\ell} \vdash \overline{\operatorname{con}} \leadsto \Sigma^{\prime}}{\Sigma \vdash\left(\text { data D } \Delta: \text { Set }_{\ell} \text { where } \overline{\text { con }}\right) \sim \Sigma^{\prime}} \\
\frac{\Sigma \vdash \Delta \quad\left(\Sigma, \text { record R } \Delta: \text { Set }_{\ell}\right) \mid \text { self }: \mathrm{R} \Delta: \text { Set }_{\ell} \vdash \overline{f l d} \leadsto \Sigma^{\prime}}{\Sigma \vdash\left(\text { record self }: \mathrm{R} \Delta: \text { Set }_{\ell} \text { where } \overline{f l d}\right) \sim \Sigma^{\prime}} \\
\frac{\Sigma \vdash A \quad(\Sigma, \text { definition } \mathrm{f}: A) \vdash P \mid \mathrm{f}:=Q: A \leadsto \Sigma^{\prime}}{\Sigma \vdash(\text { definition } \mathrm{f}: A \text { where } P) \sim \Sigma^{\prime}}
\end{gathered}
$$

$\Sigma \mid \mathrm{D} \Delta:$ Set $_{\ell} \vdash \overline{\mathrm{con}} \leadsto \Sigma^{\prime} \quad$ Presupposes: $\vdash \Sigma$ and $\Sigma \vdash \mathrm{D}: \Delta \rightarrow$ Set $_{\ell} . \quad$ Entails: $\vdash \Sigma^{\prime}$.

\begin{tabular}{|c|c|}
\hline & $\begin{array}{c}\Sigma ; \Delta(\text { self }: \mathrm{R} \hat{\Delta}) \vdash A: \text { Set }_{\ell^{\prime}} \quad \ell^{\prime} \leq \ell \\
(\Sigma, \text { projection self }: \mathrm{R} \Delta \vdash . \pi: A) \mid \text { self }: \mathrm{R} \Delta: \text { Set }_{\ell} \vdash \overline{\text { fld }} \leadsto \Sigma^{\prime}\end{array}$ \\
\hline self: R $\Delta:$ Set $_{\ell} \vdash \epsilon \leadsto \Sigma$ & $\operatorname{Set}_{\ell} \vdash \pi: A, \overline{f l d} \leadsto \Sigma^{\prime}$ \\
\hline
\end{tabular}

$\overline{\Sigma \mid \mathrm{D} \Delta: \operatorname{Set}_{\ell} \vdash \epsilon \sim \Sigma} \frac{\Sigma ; \Delta r_{\ell} \Delta_{\mathrm{c}} \quad\left(\Sigma, \text { constructor c } \Delta_{\mathrm{c}}: \mathrm{D} \Delta\right) \mid \mathrm{D} \Delta: \operatorname{Set}_{\ell} \vdash \overline{\operatorname{con}} \leadsto \Sigma^{\prime}}{\Sigma \mid \mathrm{D} \Delta: \operatorname{Set}_{\ell} \vdash \mathrm{c} \Delta_{\mathrm{c}}, \overline{\operatorname{con}} \leadsto \Sigma^{\prime}}$

$\Sigma \mid$ self: $\mathrm{R} \Delta:$ Set $_{\ell} \vdash \overline{\mathrm{fld}} \leadsto \Sigma^{\prime} \quad$ Presupposes: $\vdash \Sigma$ and $\Sigma \vdash \mathrm{R}: \Delta \rightarrow$ Set $_{\ell} . \quad$ Entails: $\vdash \Sigma^{\prime}$.

Fig. 10. Rules for checking declarations of data types, record types, and defined symbols.

\section{ELABORATION}

In the previous two sections, we have described a core language with inductive datatypes, coinductive records, identity types, and functions defined by well-typed case trees. On the other hand, we also have a surface language consisting of declarations of datatypes, record types, and functions by dependent (co)pattern matching. In this section we show how to elaborate a program in this surface language to a well-formed signature in the core language.

The main goal of this section is to describe the elaboration of a definition given by a set of (unchecked) clauses to a well-typed case tree, and prove that this translation (if it succeeds) preserves the first-match semantics of the given clauses. Before we dive into this, we first describe the elaboration for data and record types.

\subsection{Elaborating Data and Record Types}

Figure 10 gives the rules for checking declarations, constructors and projections. These rules are designed to correspond closely to those for signature extension in Fig. 5. Consequentially, if $\vdash \Sigma$ and $\Sigma \vdash \operatorname{decl} \leadsto \Sigma^{\prime}$, then also $\vdash \Sigma^{\prime}$.

\subsection{From Clauses to a Case Tree}

In Section 2 we showed how our elaboration algorithm works in a number of examples, here we describe it in general. The inputs to the algorithm are the following: 
- A signature $\Sigma$ containing previous declarations, as well as clauses for the branches of the case tree that have already been checked.

- A context $\Gamma$ containing the types of the pattern variables: $\operatorname{dom}(\Gamma)=\operatorname{PV}(\bar{q})$.

- The function $\mathrm{f}$ currently being checked.

- The copatterns $\bar{q}$ for the current branch of the case tree.

- The refined target type $C$ of the current branch.

- The user input $P$, which is described below.

The outputs of the algorithm are a signature $\Sigma^{\prime}$ extending $\Sigma$ with new clauses and a well-typed case tree $Q$ such that $\Sigma ; \Gamma \vdash \mathrm{f} \bar{q}:=Q: C \leadsto \Sigma^{\prime}$.

We represent the user input $P$ to the algorithm as an (ordered) list of partially decomposed clauses, called a left-hand side problem or lhs problem for short. Each partially decomposed clause is of the form $[E] \bar{q} \hookrightarrow r h s$ where $E$ is an (unordered) set of constraints $\left\{w_{k} / ? p_{k}: A_{k} \mid k=1 \ldots l\right\}$ between a pattern $p_{k}$ and a term $w_{k}, \bar{q}$ is a list of copatterns, and $r h s$ is a right-hand side. In the special case $E$ is empty, we have a complete clause written as $\bar{q} \hookrightarrow r h s$.

Elaboration of an lhs problem to a well-typed case tree $\Sigma ; \Gamma \vdash P \mid \mathrm{f} \bar{q}:=Q: C \sim \Sigma^{\prime}$ is defined in Fig. 11. This judgement is designed as an algorithmic version of the typing judgement for case trees $\Sigma ; \Gamma \vdash f \bar{q}:=Q: C \leadsto \Sigma^{\prime}$, where the extra user input $P$ guides the construction of the case tree. Each of the rules in Fig. 11 is a refined version of one of the rules in Fig. 8, so any case tree produced by this elaboration is well-typed by construction.

To check a definition of $\mathrm{f}: A$ with clauses $\bar{q}_{i} \hookrightarrow r h s_{i}$ for $i=1 \ldots n$, the algorithm starts with $\Gamma=\epsilon, u=\mathrm{f}$, and $P=\left\{\bar{q}_{i} \hookrightarrow r h s_{i} \mid i=1 \ldots n\right\}$. If we obtain $\Sigma ; \Gamma \vdash P \mid \mathrm{f}:=Q: A \leadsto \Sigma^{\prime}$, then the function $f$ can be implemented using the case tree $Q$.

During elaboration, the algorithm maintains the invariants that $\vdash \Sigma$ is a well-formed signature, $\Sigma \vdash \Gamma$ is a well-formed context, and $\Sigma ; \Gamma \vdash \mathrm{f}\lceil\bar{q}\rceil: C$. It also maintains the invariant that for each constraint $w_{k} / ? p_{k}: A_{k}$ in the lhs problem, we have $\Sigma ; \Gamma \vdash w_{k}: A_{k}$.

The rules for $\Sigma ; \Gamma \vdash P \mid \mathrm{f} \bar{q}:=Q: C \leadsto \Sigma^{\prime}$ make use of some auxiliary operations for manipulating lhs problems:

- After each step, the algorithm uses $\Sigma ; \Gamma \vdash E \Rightarrow \operatorname{solved}(\sigma)$ (Fig. 12) to check if the first user clause has no more (co)patterns, and all its constraints are solved. If this is the case, it returns a substitution $\sigma$ assigning a well-typed value to each of the user-written pattern variables.

- After introducing a new variable, the algorithm uses $P(x: A)$ (Fig. 13) to remove the first application pattern from each of the user clauses and to introduce a new constraint between the variable and the pattern.

- After a copattern split on a record type, the algorithm uses $P . \pi$ (Fig. 14) to partition the clauses in the lhs problem according to the projection they belong to.

- After a case split on a datatype or an equality proof, the algorithm uses $\Sigma \vdash P \sigma \Rightarrow P^{\prime}$ (Fig. 15) to refine the constraints in the problem. It uses judgements $\Sigma \vdash v / ? p: A \Rightarrow E_{\perp}$ and $\Sigma \vdash \bar{v} / ? \bar{p}: \Delta \Rightarrow E_{\perp}$ (Fig. 16) to simplify the constraints if possible, and to filter out the clauses that definitely do not match the current branch.

- To check an absurd pattern $\emptyset$, the algorithm uses $\Sigma ; \Gamma \vdash \emptyset: A$ (Fig. 17) to ensure that the type of the pattern is a caseless type [Goguen et al. 2006], i.e. a type that is empty and cannot even contain constructor-headed terms in an open context. Our language has two kinds of caseless types: datatypes $\mathrm{D} \bar{v}$ with no constructors, and identity types $u \equiv_{A} v$ where $\Sigma ; \Gamma \vdash_{x} u=? v: A \Rightarrow$ No. 
$\Sigma ; \Gamma \vdash P \mid \mathrm{f} \bar{q}:=Q: C \leadsto \Sigma^{\prime} \quad$ In all rules $P=\left\{\left[E_{i}\right] \bar{q}_{i} \hookrightarrow r h s_{i} \mid i=1 \ldots m\right\}$.

Presupposes: $\Sigma ; \Gamma \vdash f\lceil\bar{q}\rceil: C$ and $\operatorname{dom}(\Gamma)=\operatorname{PV}(\bar{q}) . \quad$ Entails: $\Sigma ; \Gamma \vdash f \bar{q}:=Q: C \leadsto \Sigma^{\prime}$.

$$
\begin{aligned}
& \frac{\bar{q}_{1}=\epsilon \quad \Sigma ; \Gamma \vdash E_{1} \Rightarrow \operatorname{solved}(\sigma) \quad r h s_{1}=v \quad \Sigma ; \Gamma \vdash v \sigma: C}{\Sigma ; \Gamma \vdash P \mid \mathrm{f} \bar{q}:=v \sigma: C \leadsto \Sigma, \text { clause } \Gamma \vdash f \bar{q} \hookrightarrow v \sigma: C} \text { Done } \\
& \frac{\bar{q}_{1}=p \bar{q}_{1}^{\prime} \quad \Sigma \vdash C \searrow(x: A) \rightarrow B \quad \Sigma ; \Gamma(x: A) \vdash P(x: A) \mid \mathrm{f} \bar{q} x:=Q: B \leadsto \Sigma^{\prime}}{\Sigma ; \Gamma \vdash P \mid \mathrm{f} \bar{q}:=\lambda x . Q: C \leadsto \Sigma^{\prime}} \text { InTro } \\
& \bar{q}_{1}=. \pi_{\mathrm{i}} \bar{q}_{1}^{\prime} \quad \Sigma \vdash C \searrow \mathrm{R} \bar{v} \quad \text { record self: } \mathrm{R} \Delta: \text { Set }_{\ell} \text { where } \overline{\pi_{\mathrm{i}}: A_{i}} \in \Sigma_{0} \\
& \frac{\left(\Sigma_{i-1} ; \Gamma \vdash P . \pi_{\mathrm{i}} \mid \mathrm{f} \bar{q} \cdot \pi_{\mathrm{i}}:=Q_{i}: A_{i}\left[\bar{v} / \Delta, \mathrm{f}\lceil\bar{q}\rceil / \text { self } \sim \Sigma_{i}\right)_{i=1 \ldots n}\right.}{\Sigma ; \Gamma \vdash P \mid \mathrm{f} \bar{q}:=\operatorname{record}\left\{\pi_{1} \mapsto Q_{1} ; \ldots ; \pi_{\mathrm{n}} \mapsto Q_{n}\right\}: C \sim \Sigma_{n}} \text { Cosplit } \\
& \bar{q}_{1}=\emptyset \quad m=1 \quad \Sigma \vdash C \searrow \mathrm{R} \bar{v} \quad \text { record }_{-}: \mathrm{R} \Delta: \text { Set }_{\ell} \text { where } \epsilon \in \Sigma \\
& r h s_{1}=\text { impossible } \\
& \Sigma ; \Gamma \vdash P \mid \mathrm{f} \bar{q}:=\operatorname{record}\{\}: C \sim \Sigma \\
& \left(x /{ }^{3} \mathrm{c}_{\mathrm{j}} \bar{p}: A\right) \in E_{1} \quad \Sigma \vdash A \searrow \mathrm{D} \bar{v} \quad \Gamma=\Gamma_{1}(x: A) \Gamma_{2} \\
& \text { data D } \Delta: \text { Set }_{\ell} \text { where } \overline{\mathrm{c}_{\mathrm{i}} \Delta_{i}} \in \Sigma_{0}
\end{aligned}
$$

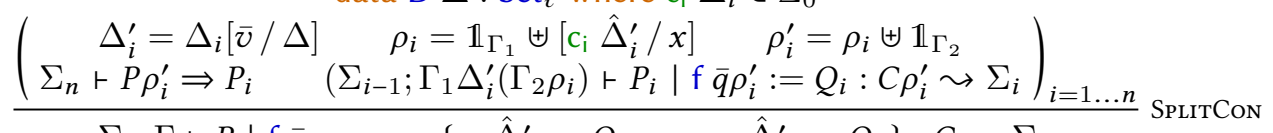

$$
\begin{aligned}
& \Sigma_{0} ; \Gamma \vdash P \mid \mathrm{f} \bar{q}:=\operatorname{case}_{x}\left\{\mathrm{c}_{1} \hat{\Delta}_{1}^{\prime} \mapsto Q_{1} ; \ldots ; \mathrm{c}_{\mathrm{n}} \hat{\Delta}_{n}^{\prime} \mapsto Q_{n}\right\}: C \sim \Sigma_{n} \\
& (x / ? \operatorname{refl}: A) \in E_{1} \quad \Sigma \vdash A \searrow u \equiv_{B} v \quad \Gamma=\Gamma_{1}(x: A) \Gamma_{2} \\
& \Sigma ; \Gamma_{1} \vdash_{x} u={ }^{?} v: B \Rightarrow \operatorname{yes}\left(\Gamma_{1}^{\prime}, \rho, \tau\right) \quad \rho^{\prime}=\rho \uplus \mathbb{1}_{\Gamma_{2}} \quad \tau^{\prime}=\tau \uplus \mathbb{1}_{\Gamma_{2}} \\
& \begin{array}{c}
\Sigma \vdash P \rho^{\prime} \Rightarrow P^{\prime} \quad \Sigma ; \Gamma_{1}^{\prime}\left(\Gamma_{2} \rho\right) \vdash P^{\prime} \mid \mathrm{f} \bar{q} \rho^{\prime}:=Q: C \rho^{\prime} \leadsto \Sigma^{\prime} \\
\Sigma ; \Gamma \vdash P \mid \mathrm{f} \bar{q}:=\text { case }_{x}\left\{\operatorname{refl}^{\tau^{\prime}} Q\right\}: C \sim \Sigma^{\prime}
\end{array} \text { SpLitEQ } \\
& \frac{(x / ? \emptyset: A) \in E_{1} \quad \Sigma ; \Gamma \vdash \emptyset: A \quad r h s_{1}=\text { impossible }}{\Sigma ; \Gamma \vdash P \mid \mathrm{f} \bar{q}:=\operatorname{case}_{x}\{\}: C \sim \Sigma} \text { SplitEmpty }
\end{aligned}
$$

Fig. 11. Rules for checking a list of clauses and elaborating them to a well-typed case tree.

$$
\Sigma ; \Gamma \vdash E \Rightarrow \operatorname{sOLVED}(\sigma)
$$

$$
\frac{\left(\Sigma \vdash\left[w_{k} / p_{k}\right] \searrow \sigma_{k}\right)_{k=1 \ldots n} \quad \sigma=\biguplus_{k} \sigma_{k} \quad\left(\Sigma ; \Gamma \vdash\left\lceil p_{k}\right\rceil \sigma=w_{k}: A_{k}\right)_{k=1 \ldots n}}{\Sigma ; \Gamma \vdash\left\{w_{k} / ? p_{k}: A_{k} \mid k=1 \ldots n\right\} \Rightarrow \operatorname{soLved}(\sigma)}
$$

Fig. 12. Rule for constructing the final substitution and checking all constraints when splitting is done.

The following rules constitute the elaboration algorithm $\Sigma ; \Gamma \vdash P \mid \mathrm{f} \bar{q}:=Q: C \leadsto \Sigma^{\prime}$ :

Done applies when the first user clause in $P$ has no more copatterns and all its constraints are solved according to $\Sigma ; \Gamma \vdash E \Rightarrow \operatorname{solved}(\sigma)$. If this is the case, then construction of the case tree is finished, adding the clause clause $\Gamma \vdash \mathrm{f} \bar{q} \hookrightarrow v \sigma: C$ to the signature. 
$P(x: A) \quad$ Replace the first application pattern $p$ in each clause by the constraint $x /{ }^{?} p: A$.

$$
\begin{aligned}
\epsilon(x: A) & =\epsilon \\
([E] p \bar{q} \hookrightarrow r h s, P)(x: A) & =([E \cup\{x / ? p: A\}] \bar{q} \hookrightarrow r h s), P(x: A)
\end{aligned}
$$

Fig. 13. Partially decomposed clauses after introducing a new variable (partial function).

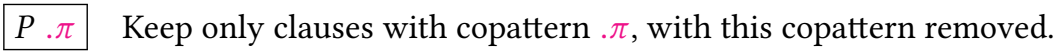

$$
\begin{array}{rlrl}
\epsilon \cdot \pi & =\epsilon \\
([E] \cdot \pi \bar{q} \hookrightarrow r h s, P) \cdot \pi & =([E] \bar{q} \hookrightarrow r h s), P . \pi & \\
\left([E] \cdot \pi^{\prime} \bar{q} \hookrightarrow r h s, P\right) \cdot \pi & =P \cdot \pi & \text { if } \pi \neq \pi^{\prime}
\end{array}
$$

Fig. 14. Partially decomposed clauses after a copattern split (partial function).

$\Sigma \vdash P \sigma \Rightarrow P^{\prime}$ ( $\Sigma$ fixed, dropped from rules.)

$$
\begin{gathered}
\frac{}{\epsilon \sigma \Rightarrow \epsilon} \quad \frac{(v / ? p: A) \in E \quad v \sigma / ? p: A \sigma \Rightarrow \perp \quad P \sigma \Rightarrow P^{\prime}}{([E] \bar{q} \hookrightarrow r h s, P) \sigma \Rightarrow P^{\prime}} \\
\frac{E=\left\{w_{k} /^{?} p_{k}: A_{k} \mid k=1 \ldots n\right\} \quad\left(w_{k} \sigma / ? p_{k}: A_{k} \sigma \Rightarrow E_{i}\right)_{k=1 \ldots n} \quad P \sigma \Rightarrow P^{\prime}}{([E] \bar{q} \hookrightarrow r h s, P) \sigma \Rightarrow\left(\left[\bigcup_{i} E_{i}\right] \bar{q} \hookrightarrow r h s\right), P^{\prime}}
\end{gathered}
$$

Fig. 15. Rules for transforming partially decomposed clauses after refining the pattern with a case split.

$$
\begin{aligned}
& \Sigma \vdash v / ? p: A \Rightarrow E_{\perp} \text { and } \Sigma \vdash \bar{v} / ? \bar{p}: \Delta \Rightarrow E_{\perp} \text { ( } \Sigma \text { fixed, dropped from rules) } \\
& \frac{v \searrow \mathrm{c} \bar{v} \quad A \searrow \mathrm{D} \bar{u} \quad \text { constructor с } \Delta_{\mathrm{c}}: \mathrm{D} \Delta \in \Sigma \quad \bar{v} / ? \bar{p}: \Delta_{\mathrm{c}}[\bar{u} / \Delta] \Rightarrow E_{\perp}}{v /{ }^{?} \mathrm{c} \bar{p}: A \Rightarrow E_{\perp}} \\
& \frac{v \searrow \operatorname{refl} A \searrow u \equiv_{B} u^{\prime}}{v / ? \operatorname{refl}: A \Rightarrow\{\}} \quad \frac{v \searrow \mathrm{c}^{\prime} \bar{v} \quad \mathrm{c} \neq \mathrm{c}^{\prime}}{v /{ }^{?} \mathrm{c} \bar{p}: A \Rightarrow \perp} \quad \frac{}{v /{ }^{?} p: A \Rightarrow\{v / ? p: A\}} \\
& \overline{\epsilon / ?} \epsilon: \epsilon \Rightarrow\{\} \quad \frac{v / ? p: A \Rightarrow E_{\perp} \quad \bar{v} / ? \bar{p}: \Delta[v / x] \Rightarrow E_{\perp}^{\prime}}{v \bar{v} / ? p \bar{p}:(x: A) \Delta \Rightarrow E_{\perp} \cup E_{\perp}^{\prime}}
\end{aligned}
$$

Fig. 16. Rules for simplifying the constraints of a partially decomposed clause.

$\Sigma ; \Gamma \vdash \emptyset: A(\Sigma$ fixed, dropped from rules $)$

$$
\frac{A \searrow \mathrm{D} \bar{v} \quad \text { data } \mathrm{D} \Delta: \text { Set }_{\ell} \text { where } \epsilon \in \Sigma}{\Gamma \vdash \emptyset: A} \quad \frac{A \searrow u \equiv_{B} v \quad \Gamma \vdash_{x} u=? v: B \Rightarrow \text { No }}{\Gamma \vdash \emptyset: A}
$$

Fig. 17. Rules for caseless types. 
INTRO applies when $C$ is a function type and all the user clauses have at least one application copattern. It constructs the case tree $\lambda x$. $Q$, using $P(x: A)$ to construct the subtree $Q$.

Cosplit applies when $C$ is a record type and all the user clauses have at least one projection copattern. It constructs the case tree record $\left\{\pi_{1} \mapsto Q_{1} ; \ldots ; \pi_{\mathrm{n}} \mapsto Q_{n}\right\}$, using $P . \pi_{\mathrm{i}}$ to construct the branch $Q_{i}$ corresponding to projection . $\pi_{\mathrm{i}}$.

CosplitEmpty applies when $C$ is a record type with no projections and the first clause starts with an absurd pattern. It then constructs the case tree record \{\} .

SPLITCON applies when the first clause has a constraint of the form $x /{ }^{?} c_{j} \bar{p}$ and the type of $x$ in $\Gamma$ is a datatype. For each constructor $c_{i}$ of this datatype, it constructs a pattern substitution $\rho_{i}$ replacing $x$ by $\mathrm{c}_{\mathrm{i}}$ applied to fresh variables. It then constructs the case tree case $_{x}\left\{\mathrm{c}_{1} \hat{\Delta}_{1}^{\prime} \mapsto Q_{1} ; \ldots ; \mathrm{c}_{\mathrm{n}} \hat{\Delta}_{n}^{\prime} \mapsto Q_{n}\right\}$, using $\Sigma \vdash P \rho_{i} \Rightarrow P_{i}$ to construct the branches $Q_{i}$.

SPLitEQ applies when the first clause has a constraint of the form $x /$ ? refl and the type of $x$ in $\Gamma$ is an identity type $u \equiv_{A} v$. It tries to unify $u$ with $v$, expecting a positive success. If unification succeeds with output $\left(\Gamma_{1}^{\prime}, \rho, \tau\right)$, it constructs the case tree case $x$ \{refl $\left.\mapsto \tau^{\tau^{\prime}} Q\right\}$, using $\Sigma \vdash P \rho^{\prime} \Rightarrow P^{\prime}$ to construct the subtree $Q$. Here $\rho^{\prime}$ and $\tau^{\prime}$ are lifted versions of $\rho$ and $\tau$ over the part of the context that is untouched by unification.

SPLitEmpty applies when the first clause has a constraint of the form $x / ?$ ? and the type of $x$ is a caseless type according to $\Sigma ; \Gamma \vdash \emptyset: A$. It then produces the case tree case $_{x}\{\}$.

Remark 23 (Limitations). The algorithm does not detect unreachable clauses, we left that aspect out of the formal description. Further, SplitEmpтy may leave some user patterns uninspected, which may then be ill-typed. However, an easy check whether the whole lhs $f\lceil\bar{q}\rceil$ is well-typed as a term can rule out ill-typed patterns.

\subsection{Preservation of First-Match Semantics}

Now that we have described the elaboration algorithm from a list of clauses to a well-typed case tree, we can state and prove our main correctness theorem. We already know that elaboration always produces a well-typed case tree by construction (if it succeeds), and that well-typed case trees are type preserving (Theorem 19) and cover all cases (Theorem 22). Now we prove that the case tree we get is the right one, i.e. that it corresponds to the definition written by the user.

To prove this theorem, we assume that the clauses we get from the user have already been scope checked, i.e. each variable in the right-hand side of a clause is bound somewhere in the patterns on the left.

Definition 24. A partially decomposed clause $[E] \bar{q} \hookrightarrow v$ is well-scoped if every free variable in $v$ occurs at least once as a pattern variable in either $\bar{q}$ or in $p$ for some constraint $(w / ? p: A) \in E$.

Theorem 25. Let $P=\left\{\bar{q}_{i} \hookrightarrow r h s_{i} \mid i=1 \ldots n\right\}$ be a problem consisting of well-scoped clauses such that $\Sigma_{0} \vdash P \mid \mathrm{f}:=Q: C \sim \Sigma$ and let $\Sigma ; \Gamma \mid \mathrm{f}: C \vdash \bar{e}: B$ be eliminations. Let index $i$ be such that:

- $\Sigma \vdash\left[\bar{e} / \bar{q}_{j}\right] \searrow \perp$ for $j=1 \ldots i-1$.

- $\Sigma \vdash\left[\bar{e} / \bar{q}_{i}\right] \searrow \sigma$.

Then $r h s_{i}=u_{i}$ is not impossible and $\Sigma \vdash Q[] \mathrm{f} \bar{e} \longrightarrow u_{i} \sigma$.

For the proof, we first need two basic properties of the auxiliary judgement $\Sigma \vdash v /{ }^{?} p: A \Rightarrow E$.

Lemma 26. If $\Sigma \vdash v / ? p: A \Rightarrow E$ where $E=\left\{w_{k} / ? p_{k}: B_{k} \mid k=1 \ldots l\right\}$, then for any substitution $\sigma$ we also have $\Sigma \vdash v \sigma / ? p: A \sigma \Rightarrow E^{\prime}$ where $E^{\prime}=\left\{w_{k} \sigma /{ }^{?} p_{k}: B_{k} \sigma \mid k=1 \ldots l\right\}$.

Proof. This follows directly from the rules of matching (Fig. 7) and simplification (Fig. 16).

Lemma 27. Let $\sigma$ be a substitution and suppose $\Sigma \vdash v / ? p: A \Rightarrow$ E. Then the following hold: 
- $\Sigma \vdash[v \sigma / p] \searrow \sigma^{\prime}$ if and only if for each $\left(w_{k} / ? p_{k}: A_{k}\right) \in E$, we have $\Sigma \vdash\left[w_{k} \sigma / p_{k}\right] \searrow \sigma_{k}$, and $\sigma^{\prime}=\biguplus_{k} \sigma_{k}$.

- $\Sigma \vdash[v \sigma / p] \searrow \perp$ if and only if for some $\left(w_{k} / ? p_{k}: A_{k}\right) \in E$, we have $\Sigma \vdash\left[w_{k} \sigma / p_{k}\right] \searrow \perp$.

Proof. This follows directly from the rule of matching (Fig. 7) and simplification (Fig. 16).

The following lemma is the main component of the proof. It generalizes the statement of Theorem 25 to the case where the left-hand side has already been refined to $f \bar{q}$ and the user clauses have been partially decomposed. From this lemma, the main theorem follows directly by taking $\bar{q}=\epsilon$ and $E_{i}=\{\}$ for $i=1 \ldots n$.

Lemma 28. Let $P=\left\{\left[E_{i}\right] \bar{q}_{i} \hookrightarrow r h s_{i} \mid i=1 \ldots n\right\}$ be a list of well-scoped partially decomposed clauses such that $\Sigma_{0} ; \Gamma_{0} \vdash P \mid \mathrm{f} \bar{q}:=Q: C \leadsto \Sigma$, and suppose $\Gamma \vdash \sigma_{0}: \Gamma_{0}$ and $\Sigma ; \Gamma \mid \mathrm{f} \bar{q} \sigma_{0}$ : $C \sigma_{0} \vdash \bar{e}: B$. If there is an index $i$ such that:

- For each $j=1 \ldots i-1$ and each constraint $\left(w_{k} / ? p_{k}: A_{k}\right) \in E_{j}$, either $\Sigma \vdash\left[w_{k} \sigma_{0} / p_{k}\right] \searrow \theta_{j k}$ or $\Sigma \vdash\left[w_{k} \sigma_{0} / p_{k}\right] \searrow \perp$.

- For each $j=1 \ldots i-1$, either $\Sigma \vdash\left[\bar{e} / \bar{q}_{j}\right] \searrow \theta_{j 0}$ or $\Sigma \vdash\left[\bar{e} / \bar{q}_{j}\right] \searrow \perp$.

- For each $j=1 \ldots i-1$, either $\Sigma \vdash\left[w_{k} \sigma_{0} / p_{k}\right] \searrow \perp$ for some constraint $\left(w_{k} /{ }^{?} p_{k}: A_{k}\right) \in E_{j}$, or $\Sigma \vdash\left[\bar{e} / \bar{q}_{j}\right] \searrow \perp$.

- For each $\left(w_{k} / ? p_{k}: A_{k}\right) \in E_{i}$, we have $\Sigma \vdash\left[w_{k} \sigma_{0} / p_{k}\right] \searrow \theta_{k}$.

- $\Sigma \vdash\left[\bar{e} / \bar{q}_{i}\right] \searrow \theta_{0}$.

Then $r h s_{i}=v_{i}$ is not impossible and $\Sigma \vdash Q \sigma_{0} \bar{e} \longrightarrow v_{i} \theta$ where $\theta=\theta_{0} \uplus\left(\biguplus_{k} \theta_{k}\right)$.

Proof. By induction on the derivation of $\Sigma_{0} ; \Gamma_{0} \vdash P \mid \mathrm{f} \bar{q}:=Q: C \leadsto \Sigma$.

\section{RELATED WORK}

Dependent pattern matching was introduced in the seminal work by Coquand [1992]. It is used in the implementation of various dependently typed languages such as Agda [Norell 2007], Idris [Brady 2013], the Equations package for Coq [Sozeau 2010], and Lean [de Moura et al. 2015].

Previous work by Norell [2007], Sozeau [2010], and Cockx [2017] also describe elaborations from clauses to a case tree, but in much less detail than presented here, and they do not support copatterns or provide a correctness proof. In cases where both our current algorithm and these previous algorithms succeed, we expect there is no difference between the resulting case trees. However, our current algorithm is much more flexible in the placement of dot patterns, so it accepts more definitions than was possible before (see Example 4).

The translation from a case tree to primitive datatype eliminators was pioneered by McBride [2000] and further detailed by Goguen et al. [2006] for type theory with uniqueness of identity proofs and Cockx [2017] in a theory without.

Forced patterns, as well as forced constructors, were introduced by Brady et al. [2003]. Brady et al. focus mostly on the compilation process and the possibility to erase arguments and constructor tags, while we focus more on the process of typechecking a definition by pattern matching and the construction of a case tree.

Copatterns were introduced in the simply-typed setting by Abel et al. [2013] and subsequently used for unifying corecursion and recursion in System $\mathrm{F}^{\omega}$ [Abel and Pientka 2013]. In the context of Isabelle/HOL, Blanchette et al. [2017] use copatterns as syntax for mixed recursive-corecursive definitions. Setzer et al. [2014] give an algorithm for elaborating a definition by mixed (co)pattern matching to a nested case expression, yet only for a simply typed language. Thibodeau et al. [2016] present a language with deep (co)pattern matching and a restricted form of dependent types. In their language, types can only depend on a user-defined domain with decidable equality and the 
types of record fields cannot depend on each other, thus, a self value is not needed for checking projections. They feature indexed data and record types in the surface language which are elaborated into non-indexed types via equality types, just as in our core language.

The connection between focusing [Andreoli 1992] and pattern matching has been systematically explored by Zeilberger [2009]. In Licata et al. [2008] copatterns ("destructor patterns") also appear in the context of simple typing with connectives from linear logic. Krishnaswami [2009] boils the connection to focusing down to usual non-linear types; however, he has no copatterns as he only considers the product type as multiplicative (tensor), not additive. Thibodeau et al. [2016] extend the connection to copatterns for indexed record types.

Elaborating a definition by pattern matching to a case tree [Augustsson 1985] simultaneously typechecks the clauses and checks their coverage, so our algorithm has a lot in common with coverage checking algorithms. For example, Norell [2007] views the construction of a case tree as a part of coverage checking. Oury [2007] presents a similar algorithm for coverage checking and detecting useless cases in definitions by dependent pattern matching.

\section{FUTURE WORK AND CONCLUSION}

In this paper, we give a description of an elaboration algorithm for definitions by dependent copattern matching that is at the same time elegant enough to be intuitively understandable, simple enough to study formally, and detailed enough to serve as the basis for a practical implementation.

The implementation of our algorithm as part of the Agda typechecker is at the moment of writing still work in progress. In fact, the main reason to write this paper was to get a clear idea of what exactly should be implemented. For instance, while working on the proof of Theorem 25, we were quite surprised to discover that it did not hold at first: matching was performed lazily from left to right, but the case tree produced by elaboration may not agree on this order! This problem was not just theoretical, but also manifested itself in the implementation of Agda as a violation of subject reduction [Agda issue 2018a]. Removing the shortcut rule from the definition of matching removed this behavioral divergence mismatch. The complete formalization of the elaboration algorithm in this paper lets us continue the implementation with confidence.

Agda also has a number of features that are not described in this paper, such as nonrecursive record types with $\eta$ equality and general indexed datatypes (not just the identity type). The implementation also has to deal with the insertion of implicit arguments, the presence of metavariables in the syntax, and reporting understandable errors when the algorithm fails. Based on our practical experience, we are confident that the algorithm presented here can be extended to deal with all of these features.

\section{ACKNOWLEDGMENTS}

The authors acknowledge support by the Swedish Research Council (Vetenskapsrådet) under Grant No. 621-2014-4864 Termination Certificates for Dependently-Typed Programs and Proofs via Refinement Types. Our research group is part of the EU Cost Action CA15123 The European research network on types for programming and verification (EUTypes). We thank the anonymous referees of the draft version for their comments that helped improving the presentation. 


\section{REFERENCES}

Andreas Abel and Brigitte Pientka. 2013. Wellfounded Recursion with Copatterns: A Unified Approach to Termination and Productivity. In Proceedings of the Eighteenth ACM SIGPLAN International Conference on Functional Programming, ICFP'13, Boston, MA, USA, September 25-27, 2013, Greg Morrisett and Tarmo Uustalu (Eds.). ACM Press, 185-196. https: //doi.org/10.1145/2500365.2500591

Andreas Abel, Brigitte Pientka, David Thibodeau, and Anton Setzer. 2013. Copatterns: Programming Infinite Structures by Observations. In The 40th Annual ACM SIGPLAN-SIGACT Symposium on Principles of Programming Languages, POPL'13, Rome, Italy, fanuary 23 - 25, 2013, Roberto Giacobazzi and Radhia Cousot (Eds.). ACM Press, 27-38. http://dl.acm.org/ citation.cfm?id=2429069

Agda development team. 2017. Agda 2.5.3 documentation. http://agda.readthedocs.io/en/v2.5.3/

Agda issue. 2017a. Disambiguation of type based on pattern leads to non-unique meta solution. (2017). https://github. com/agda/agda/issues/2834 (on the Agda bug tracker).

Agda issue. 2017b. Internal error in src/full/Agda/TypeChecking/Coverage/Match.hs:312. (2017). https://github.com/agda/ agda/issues/2874 (on the Agda bug tracker).

Agda issue. 2017c. Panic: unbound variable. (2017). https://github.com/agda/agda/issues/2856 (on the Agda bug tracker)

Agda issue. 2017d. Record constructor is accepted, record pattern is not. (2017). https://github.com/agda/agda/issues/2850 (on the Agda bug tracker).

Agda issue. 2018a. Mismatch between order of matching in clauses and case tree; subject reduction broken. (2018). https: //github.com/agda/agda/issues/2964 (on the Agda bug tracker).

Agda issue. 2018b. Unifier throws away pattern. (2018). https://github.com/agda/agda/issues/2896 (on the Agda bug tracker).

Jean-Marc Andreoli. 1992. Logic Programming with Focusing Proofs in Linear Logic. fournal of Logic and Computation 2 , 3 (1992), 297-347. https://doi.org/10.1093/logcom/2.3.297

Lennart Augustsson. 1985. Compiling Pattern Matching. In Functional Programming Languages and Computer Architecture, FPCA 1985, Nancy, France, September 16-19, 1985, Proceedings (Lecture Notes in Computer Science), Jean-Pierre Jouannaud (Ed.), Vol. 201. Springer, 368-381. https://doi.org/10.1007/3-540-15975-4_48

Jasmin Christian Blanchette, Aymeric Bouzy, Andreas Lochbihler, Andrei Popescu, and Dmitriy Traytel. 2017. Friends with Benefits - Implementing Corecursion in Foundational Proof Assistants. In Programming Languages and Systems - 26th European Symposium on Programming, ESOP 2017, Held as Part of the European foint Conferences on Theory and Practice of Software, ETAPS 2017, Uppsala, Sweden, April 22-29, 2017, Proceedings (Lecture Notes in Computer Science), Hongseok Yang (Ed.), Vol. 10201. Springer, 111-140. https://doi.org/10.1007/978-3-662-54434-1_5

Edwin Brady. 2013. Idris, a general-purpose dependently typed programming language: Design and implementation. fournal of Functional Programming 23, 5 (2013), 552-593. https://doi.org/10.1017/S095679681300018X

Edwin Brady, Conor McBride, and James McKinna. 2003. Inductive Families Need Not Store Their Indices. In Types for Proofs and Programs, International Workshop, TYPES 2003, Torino, Italy, April 30 - May 4, 2003, Revised Selected Papers (Lecture Notes in Computer Science), Stefano Berardi, Mario Coppo, and Ferruccio Damiani (Eds.), Vol. 3085. Springer, 115-129. https://doi.org/10.1007/978-3-540-24849-1_8

Luca Cardelli. 1984. Compiling a Functional Language. In Proceedings of the 1984 ACM Conference on LISP and Functional Programming, August 5-8, 1984, Austin, Texas, USA. ACM Press, 208-217. http://lucacardelli.name/Papers/CompilingML. A4.pdf

Jesper Cockx. 2017. Dependent pattern matching and proof-relevant unification. Ph.D. Dissertation. KU Leuven.

Jesper Cockx, Dominique Devriese, and Frank Piessens. 2016. Unifiers as equivalences: proof-relevant unification of dependently typed data, See [Garrigue et al. 2016], 270-283. https://doi.org/10.1145/2951913.2951917

Thierry Coquand. 1992. Pattern Matching with Dependent Types. In Proceedings of the 1992 Workshop on Types for Proofs and Programs, Båstad, Sweden, June 1992, Bengt Nordström, Kent Pettersson, and Gordon Plotkin (Eds.). 71-83. http: //www.cse.chalmers.se/ coquand/pattern.ps

Leonardo Mendonça de Moura, Soonho Kong, Jeremy Avigad, Floris van Doorn, and Jakob von Raumer. 2015. The Lean Theorem Prover (System Description). In Automated Deduction - CADE-25 - 25th International Conference on Automated Deduction, Berlin, Germany, August 1-7, 2015, Proceedings (Lecture Notes in Computer Science), Amy P. Felty and Aart Middeldorp (Eds.), Vol. 9195. Springer, 378-388. https://doi.org/10.1007/978-3-319-21401-6_26

Jacques Garrigue, Gabriele Keller, and Eijiro Sumii (Eds.). 2016. Proceedings of the 21st ACM SIGPLAN International Conference on Functional Programming, ICFP 2016, Nara, Japan, September 18-22, 2016. ACM Press. https://doi.org/10.1145/ 2951913

Healfdene Goguen, Conor McBride, and James McKinna. 2006. Eliminating Dependent Pattern Matching. In Algebra, Meaning, and Computation, Essays Dedicated to Joseph A. Goguen on the Occasion of His 65th Birthday (Lecture Notes in Computer Science), Kokichi Futatsugi, Jean-Pierre Jouannaud, and José Meseguer (Eds.), Vol. 4060. Springer, 521-540. https://doi.org/10.1007/11780274_27 
INRIA. 2017. The Coq Proof Assistant Reference Manual (version 8.7 ed.). INRIA. http://coq.inria.fr/

Neelakantan R. Krishnaswami. 2009. Focusing on pattern matching. In Proceedings of the 36th ACM SIGPLAN-SIGACT Symposium on Principles of Programming Languages, POPL 2009, Savannah, GA, USA, fanuary 21-23, 2009, Zhong Shao and Benjamin C. Pierce (Eds.). ACM Press, 366-378. https://doi.org/10.1145/1480881.1480927

Daniel R. Licata, Noam Zeilberger, and Robert Harper. 2008. Focusing on Binding and Computation. In Proceedings of the Twenty-Third Annual IEEE Symposium on Logic in Computer Science, LICS 2008, 24-27 June 2008, Pittsburgh, PA, USA, Frank Pfenning (Ed.). IEEE Computer Society Press, 241-252. https://doi.org/10.1109/LICS.2008.48

Luc Maranget. 1992. Compiling Lazy Pattern Matching. In LISP and Functional Programming. 21-31. https://doi.org/10. 1145/141471.141499

Conor McBride. 2000. Dependently typed functional programs and their proofs. Ph.D. Dissertation. University of Edinburgh.

Ulf Norell. 2007. Towards a practical programming language based on dependent type theory. Ph.D. Dissertation. Chalmers University of Technology.

Nicolas Oury. 2007. Pattern matching coverage checking with dependent types using set approximations. In Proceedings of the ACM Workshop Programming Languages meets Program Verification, PLPV 2007, Freiburg, Germany, October 5, 2007, Aaron Stump and Hongwei Xi (Eds.). ACM Press, 47-56. https://doi.org/10.1145/1292597.1292606

Robert Pollack. 1998. How to Believe a Machine-Checked Proof. In Twenty Five Years of Constructive Type Theory, Giovanni Sambin and Jan Smith (Eds.). Oxford University Press. http://www.brics.dk/RS/97/18/BRICS-RS-97-18.pdf

Anton Setzer, Andreas Abel, Brigitte Pientka, and David Thibodeau. 2014. Unnesting of Copatterns. In Rewriting and Typed Lambda Calculi - Foint International Conference, RTA-TLCA 2014, Held as Part of the Vienna Summer of Logic, VSL 2014, Vienna, Austria, Fuly 14-17, 2014. Proceedings (Lecture Notes in Computer Science), Gilles Dowek (Ed.), Vol. 8560. Springer, 31-45. https://doi.org/10.1007/978-3-319-08918-8_3

Matthieu Sozeau. 2010. Equations: A Dependent Pattern-Matching Compiler. In Interactive Theorem Proving, First International Conference, ITP 2010, Edinburgh, UK, July 11-14, 2010. Proceedings (Lecture Notes in Computer Science), Matt Kaufmann and Lawrence C. Paulson (Eds.), Vol. 6172. Springer, 419-434. https://doi.org/10.1007/978-3-642-14052-5_29

David Thibodeau, Andrew Cave, and Brigitte Pientka. 2016. Indexed codata types, See [Garrigue et al. 2016], 351-363. https://doi.org/10.1145/2951913.2951929

Noam Zeilberger. 2008. Focusing and higher-order abstract syntax. In Proceedings of the 35th ACM SIGPLAN-SIGACT Symposium on Principles of Programming Languages, POPL 2008, San Francisco, California, USA, January 7-12, 2008, George C. Necula and Philip Wadler (Eds.). ACM Press, 359-369. https://doi.org/10.1145/1328438.1328482

Noam Zeilberger. 2009. The Logical Basis of Evaluation Order and Pattern-Matching. Ph.D. Dissertation. Carnegie Mellon University. http://software.imdea.org/ noam.zeilberger/thesis.pdf 